ARTICLE

DOI: $10.1038 /$ s41467-018-07077-1

\title{
H3K9 methyltransferases and demethylases control lung tumor-propagating cells and lung cancer progression
}

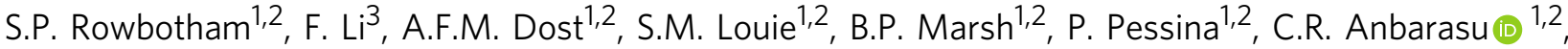 \\ C.F. Brainson ${ }^{4}$, S.J. Tuminello ${ }^{5}$, A. Lieberman ${ }^{6}$, S. Ryeom ${ }^{6}$, T.M. Schlaeger ${ }^{1}$, B.J. Aronow ${ }^{7}$, H. Watanabe ${ }^{5}$, \\ K.K. Wong ${ }^{3} \&$ C.F. Kim ${ }^{1,2,8}$
}

Epigenetic regulators are attractive anticancer targets, but the promise of therapeutic strategies inhibiting some of these factors has not been proven in vivo or taken into account tumor cell heterogeneity. Here we show that the histone methyltransferase G9a, reported to be a therapeutic target in many cancers, is a suppressor of aggressive lung tumorpropagating cells (TPCS). Inhibition of G9a drives lung adenocarcinoma cells towards the TPC phenotype by de-repressing genes which regulate the extracellular matrix. Depletion of G9a during tumorigenesis enriches tumors in TPCs and accelerates disease progression metastasis. Depleting histone demethylases represses G9a-regulated genes and TPC phenotypes. Demethylase inhibition impairs lung adenocarcinoma progression in vivo. Therefore, inhibition of G9a is dangerous in certain cancer contexts, and targeting the histone demethylases is a more suitable approach for lung cancer treatment. Understanding cellular context and specific tumor populations is critical when targeting epigenetic regulators in cancer for future therapeutic development.

\footnotetext{
${ }^{1}$ Stem Cell Program, Division of Hematology/Oncology and Pulmonary and Respiratory Diseases, Children's Hospital Boston, Boston, MA 02115, USA. 2 Department of Genetics, Harvard Medical School, Boston, MA 02115, USA. ${ }^{3}$ Laura and Isaac Perlmutter Cancer Center, New York University Langone Medical Center, New York, NY 10016, USA. ${ }^{4}$ Department of Toxicology and Cancer Biology, University of Kentucky, Lexington, KY 40536, USA.

${ }^{5}$ Department of Medicine, Division of Pulmonary, Critical Care and Sleep Medicine, Tisch Cancer Institute, Icahn School of Medicine at Mount Sinai, New York, NY 10029, USA. ${ }^{6}$ Department of Cancer Biology, Perelman School of Medicine at the University of Pennsylvania, Abramson Cancer Center, Philadelphia, PA 19104, USA. ${ }^{7}$ Division of Biomedical Informatics, Cincinnati Children's Research Foundation, University of Cincinnati College of Medicine, Cincinnati, OH 45229, USA. ${ }^{8}$ Harvard Stem Cell Institute, Cambridge, MA 02138, USA. Correspondence and requests for materials should be addressed to C.F.K. (email: carla.kim@childrens.harvard.edu)
} 
$\mathrm{T}$ umors are phenotypically heterogeneous, containing cells with widely different disease-promoting potential. The most aggressive cells exhibit regenerative and proliferative behaviors associated with tissue progenitor cells and are often referred to as cancer stem cells or tumor-propagating cells (TPCs). We previously identified TPCs in the KraLSSL $p 53$ floxfflox (hereafter referred as Kras;p53) mouse model of lung adenocarcinoma. Sca-1 (stem cell antigen-1, also Ly6a) and CD24 enriched for a population of cells with enhanced transplantation efficiency ${ }^{1}$, and greater metastatic potential ${ }^{2}$. A combination of CD24, ITGB4 (integrin $\beta 4$ ), and high Notch receptor expression has also been used to isolate TPCs from this model ${ }^{3}$. These approaches have made it possible to investigate why TPCs behave differently compared to other tumor cells.

Chromatin-modifying and/or chromatin-interacting proteins, often referred to as epigenetic regulators, play essential roles in delineating cell fate and governing the behavior of stem or progenitor cells. Epigenetic regulators are frequently amplified, mutated, or mis-regulated in cancer, often playing important roles in tumorigenesis, and have thus become the focus of an entire field of therapeutic discovery ${ }^{4,5}$. G9a and Glp (also Ehmt2 and Ehmt1, respectively) are histone methyltransferases (HMTs) that catalyze the mono-methylation and di-methylation of histone $\mathrm{H} 3$ lysine 9 (H3K9). These enzymes are known for regulating embryonic stem cell differentiation, particularly into neuronal lineages ${ }^{6,7}$, and have also been linked to cancer, where G9a is mutated or amplified at a low frequency in a variety of tumors ${ }^{8}$. The majority of studies describe G9a as an oncogene, finding G9a to have pro-proliferative and proepithelial-mesenchymal transition (pro-EMT) functions ${ }^{9-12}$. These results have spurred the development of chemical inhibitors of G9a/Glp in the hope that they could be used as anticancer therapeutics ${ }^{13-15}$. Chromatin modifications are dynamically regulated, and multiple lysine demethylases (KDMs) can remove H3K9 methylation, including Kdm3a (also Jmjd1a, JHDM2a) and $\mathrm{Phf}^{16,17}$. These enzymes have been linked to tumorigenesis and stem cell behavior in other tissues ${ }^{18-20}$, making them plausible candidates to regulate these phenotypes in the lung. However, a specific role for $\mathrm{H} 3 \mathrm{~K} 9$ methylation regulators in progenitor cell behavior in lung cancer has not been explored.

By screening for regulators of TPC surface markers, we identify the H3K9me1/2 HMTs G9a and Glp as TPC suppressors; depleting G9a during tumorigenesis expands the TPC population and accelerates disease progression. Inhibiting or depleting G9a upregulates genes associated with mutant KRAS and extracellular matrix (ECM) regulation, and depleting these genes or overexpressing G9a impedes tumor transplantation. Depleting or inhibiting H3K9me1/2 KDMs impairs the TPC phenotype, revealing this a possible means to further develop a lung adenocarcinoma therapeutic.

\section{Results}

G9a/Glp inhibition drives cells towards the TPC phenotype. To determine which molecular pathways regulate lung adenocarcinoma TPCs, we tested a library of stem cell-regulating small molecules (Table 1). We screened a TPC-like cell line, CK1750², for changes in the surface antigens Sca-1 and CD24, reasoning that changes in these markers could correspond to changes in TPC activity. Multiple compounds increased the levels of at least one TPC antigen (Fig. 1a, Supplementary Fig. 1a), and we focused on characterizing the effect of the molecule that increased Sca-1 the most significantly (1.8-fold vs. dimethyl sulfoxide (DMSO), $P=$ $0.0015, t$ test), UNC0638. UNC0638, a potent specific inhibitor of the H3K9 mono-methyltransferase and di-methyltransferases Glp and G9a ${ }^{21}$, increased Sca-1 in multiple adenocarcinoma cell lines, with a greater difference in lines with low endogenous Sca-1 levels, TM1 and TnM2 (Fig. 1b, Supplementary Fig. 1b). UNC0638 also increased Sca-1 mRNA (2.1-fold, $P=0.036,19.8$-fold, $P=0.0005$, and 104.3-fold, $P=0.0001, t$ test), implying that higher Sca-1 levels were due to upregulated transcription (Fig. 1c).

We have previously demonstrated that CD24 and Sca-1 mark a population enriched in TPCs ${ }^{1,2}$. To further explore the effects of G9a/Glp inhibition (hereafter referred as G9ai) on tumorpropagating ability, we modeled this phenotype with primary adenocarcinoma cells in Matrigel air-liquid interface cultures. Sca-1+CD24+ cells formed organoids at a higher rate than other primary tumor populations (5.8 vs. $3.1,3.3$, and $1.9 \%, P=0.002$, $P=0.0007, P=0.0002, t$ test), demonstrating that more efficient in vitro organoid formation correlates with a TPC-enriched population (Supplementary Fig. 1c). G9ai of Sca-1-low adenocarcinoma cell lines increased the proportion of Sca-1-expressing cells and led to increased organoid-forming efficiency (3.95 vs. $0.75 \%, P=0.004$ and 6.1 vs. $2.7 \%, P=0.025, t$ test) (Fig. $1 \mathrm{~d}$ ). G9ai of unsorted primary adenocarcinoma cells in 3D culture also increased organoid formation ( 0.97 vs. $0.25 \%, P=0.027,1.32$ vs. $0.29 \%, P=0.03, t$ test) (Fig. 1e) and resulted in more Sca- $1+$ cells when cultures were analyzed at the experimental endpoint (Supplementary Fig. 1d). To further demonstrate that G9ai could promote a TPC phenotype, we inhibited Sca-1-low adenocarcinoma cell lines and intravenously injected them into immunocompromised (nude) recipient mice (Fig. 1f, Supplementary Fig. 2a). At the experimental endpoint, we detected lung tumors in the recipients of both G9ai and vehicle control-treated cells (Fig. 1f, Supplementary Fig. 2b). However, mice that had received G9ai cells more frequently presented with tumors outside the lung (thoracic lymph nodes, aorta, subcutaneous) (58 vs. $17 \%$, $P=0.017$, Fisher's exact test) (Fig. 1f, Supplementary Fig. 2b), demonstrating that G9ai can also promote a more tumorigenic TPC phenotype in vivo.

Our findings that lower G9a and $\mathrm{H} 3 \mathrm{~K} 9 \mathrm{me} 2$ were associated with TPCs and that G9ai can increase the tumorigenicity of cancer cells appeared to contrast with other studies, suggesting that G9a is oncogenic ${ }^{9-12}$. Although $1 \mu \mathrm{M}$ UNC0638 (the concentration used in our assays) did not affect cell proliferation or survival (Supplementary Fig. 2c, d), higher concentrations (5 and $10 \mu \mathrm{M}$ ) resulted in significant decreases (optical density (OD) 6001.16 vs. $0.74,0.09, P=0.002,<0.001, t$ test, Supplementary Fig. 2d). This was in line with previous findings describing G9 as a pro-proliferative ${ }^{10,12}$, and shows how without considering cellular context and tumor heterogeneity, G9ai could be considered as a potential anti-oncogenic treatment.

As enzymatic inhibition of G9a/Glp could promote TPC characteristics in adenocarcinoma cells, we hypothesized that less G9a/Glp or deregulated H3K9me1/2 could be an intrinsic TPC property. Re-analysis of our previous gene expression data comparing Kras;p53 TPCs vs. non-TPCs ${ }^{2}$ indicated that G9a, but not Glp, was significantly downregulated in the TPC-enriched population (0.41-fold, $P=0.037, t$ test) (Fig. $1 \mathrm{~g}$ ), suggesting that reduced G9a levels may be important to lung TPCs. To confirm this association, we stained global H3K9me2 and Sca-1 in sorted lung adenocarcinoma populations. We found that global H3K9me2 was significantly higher in the least tumorigenic, Sca-1-CD24- cell population than in the CD24+Sca-1-, CD24-Sca-1+ and CD24 + Sca-1+ populations (36.3 fluorescent units vs. $6.4,4.8,6.2, P=$ $0.004, P=0.003$, and $P=0.004, t$ test), while Sca- 1 was significantly higher in the Sca-1+CD24+ population compared to all the others (89.5 vs. $14.0,9.6,33.4, P=0.003, P=0.003$, and $P=0.013, t$ test) (Fig. 1h, i). These data show an inverse association between Sca-1, TPC, and $\mathrm{H} 3 \mathrm{~K} 9 \mathrm{me} 2$, suggesting that $\mathrm{H} 3 \mathrm{~K} 9$ demethylation may be a feature of, or a prerequisite for, lung adenocarcinoma TPCs and tumor progression and metastasis. 


\section{Table 1 Composition of the Stem Cell Chemical Library used for screening lung adenocarcinoma TPCs}

\begin{tabular}{|c|c|c|c|}
\hline Compound & Mechanism & Compound & Mechanism \\
\hline CCPA & $\mathrm{A} 1+$ & Compound W & Notch \\
\hline SQ22536 & Acase- & DAPT & Notch \\
\hline Forskolin & Acase+ & DLPC & nr2a5 \\
\hline SR1 & $a h r-$ & 2-Phospho-L-ascorbic acid & $P$ \\
\hline ITE & $\mathrm{AHR}+$ & IPA-3 & Pak- \\
\hline ABT 702 & $\mathrm{AK}-$ & $\mathrm{EB}-47$ & parp1- \\
\hline \multicolumn{4}{|l|}{ dihydrochloride } \\
\hline KP372-1 & $\mathrm{AKT}-$ & DMPQ dihydrochloride & pdgf $r$ inh \\
\hline$(+)-J Q 1-1 \mathrm{mg}$ & BET- & SU6656 & $\mathrm{pdgf} / \mathrm{fgf} / \mathrm{vegf} \mathrm{r}$ inh \\
\hline Dorsomorphin & bmpr- & Mycophenolate mofetil & $\mathrm{PDH} 2$ \\
\hline CITCO & CAR & PS48 & PDK1 \\
\hline Indomethacin & $\operatorname{cox}$ & 16,16-Dimethyl prostaglandin E2 & PGE2 \\
\hline AMD3100 & $\operatorname{cxcr} 4-$ & GDC-0941 & pi3k \\
\hline FCCP & decoupl & PI-103 & pi3k- mtor- dnapk- \\
\hline A77 1726 & dhodh & cAMPS-Rp & PKA- \\
\hline RG 108 & DNMT & GSK 0660 & PPARd- \\
\hline $5-A z a-2^{\prime}-$ & DNMT- & BADGE & pparg- \\
\hline \multicolumn{4}{|l|}{ deoxycytidine } \\
\hline 5-Azacytidine & DNMT1- & GW9662 & PPARg- \\
\hline SGC 0946 & DOT1L- & Troglitazone & pparg+ \\
\hline AG 1478 & EGFR & AM 114 & Proteasome \\
\hline Erlotinib & egfr- & NSC 23766 & RAC \\
\hline GSK126 & $\mathrm{EZH} 2-$ & BMS 493 & $\mathrm{RAR}+$ \\
\hline PF 573228 & FAK & BMS195614 & RARa- \\
\hline PD161570 & fgfr- & AM580 & RARa+ \\
\hline UNC 0638 & g9a inh & AC 261066 & $\mathrm{RARb}+$ \\
\hline Dexamethasone & gc & CD2665 & RARbg- \\
\hline Depudecin & $\mathrm{HDAC}-$ & BMS 961 & $\mathrm{RARg}+$ \\
\hline Sodium butyrate & $\mathrm{HDAC}-$ & y27632 & rock- \\
\hline Trichostatin A & HDAC- & Blebbistatin & rock/mlck \\
\hline Valproic acid & $\mathrm{HDAC}-$ & BI-D1870 & RSK \\
\hline GANT 61 & $\mathrm{HH}-$ & W146 & s1p1- \\
\hline Purmorphamine & $\mathrm{HH}+$ & SEW2871 & s1p1+ \\
\hline $20(S)-$ & $\mathrm{HH}+$ & Splitomycin & $\operatorname{sirp} 2-$ \\
\hline \multicolumn{4}{|c|}{ Hydroxycholesterol } \\
\hline $10 \times 2$ & $\mathrm{HIF}+$ & ex527 & sirt1- \\
\hline IKK16 & IKK & agk2 & sirt2- \\
\hline Revstarol & jak $3 / 2 / 1$ inh & A83-01 & $\operatorname{tgfb}-$ \\
\hline cp690550 & jak- & repsox & $\operatorname{tgfb}-$ alk5- \\
\hline Tranylcypromine & LSD1- & IDE-1 & $\mathrm{TGFb}+$ \\
\hline \multicolumn{4}{|l|}{$\mathrm{HCl}$} \\
\hline PD98059 & MAPK & SB431542 & $\operatorname{tgfb1}$ \\
\hline Nutlin & mdm2 inh & TLR3/dsRNA complex inhibitor & tlr3 inh \\
\hline PD0325901 & MEK & MLS000389544 & $\mathrm{TR}-$ \\
\hline Rapamycin & mtor & $1-850$ & $\mathrm{TR}+$ \\
\hline Honokiol & nfkb- & 3,3',5-Triiodo-L-thyronine sodium salt & $\mathrm{TR}+$ \\
\hline Betulinic acid & $\mathrm{nfkb}+$ & RN 1734 & TRP channels \\
\hline SNAP & $\mathrm{NO}$ & ZM 306416 hydrochloride & vegf $r$ inh \\
\hline DETA NONOate & $\mathrm{NO}+$ & IWP-2 & wnt- \\
\hline \multirow[t]{2}{*}{ L-NAME } & $\mathrm{NOS}-$ & XAV 939 & Wnt- \\
\hline & & CHIR99021 & wnt+gsk- \\
\hline
\end{tabular}

G9a depletion promotes tumor progression and metastasis. Historically, TPCs have largely been studied ex vivo using surface markers and transplantation experiments. Identification of G9a as a TPC-regulating enzyme allowed us to test its function, and the role of TPCs more broadly, in tumorigenesis in situ. We utilized bi-functional Cre-U6shRNA lentiviral vectors to knockdown G9a only in the induced tumors of Kras;p53 recipient mice (Fig. 2a, Supplementary Fig. 3a). At the experimental endpoint, recipients of $s h G 9 a$ lentivirus had remarkably progressed disease compared to shLuciferase controls, with significantly increased tumor number $(P=0.0005, t$ test) and tumor burden (Fig. 2b, Supplementary Fig. 3b). Histological examination of tumors revealed that $\operatorname{sh} G 9 a$ recipients had significantly advanced disease $(P<$ $0.0001, \chi^{2}$ test), with the majority of mice presenting aggressive, poorly differentiated, grade 4 tumors, while the majority of control mice had only grade 2 tumors (Fig. 2c, Supplementary Fig. 3c). Quantitative PCR (qPCR) of isolated tumor cells and immunostains of lung tumor sections from these mice confirmed that G9a was significantly depleted in $\operatorname{sh} G 9 a$ recipient tumors compared to controls (Supplementary Fig. 3d, e). Although we sacrificed mice in the same time frame for comparison, shG9a mice grew sicker more quickly than controls $(P=0.017$, Mantel-Cox log-rank test), suggesting that G9a depletion was not simply selecting for escaped, more aggressive tumor cells (Supplementary Fig. 3f). Proliferation was not altered in endpoint shG9a tumors; similar proportions of cells stained for Ki67 in shG9a and control tumors (21.1 vs. $14.6 \%, P=0.45, t$ test) (Supplementary Fig. 4a, b). 
a

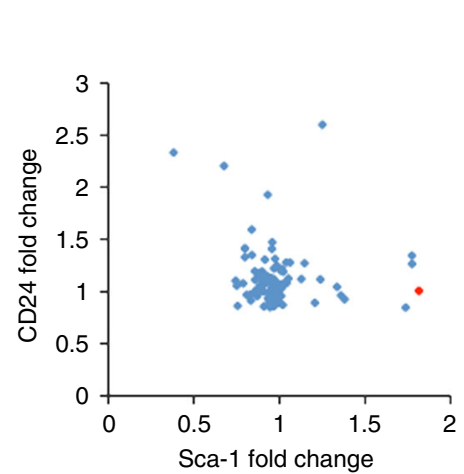

d

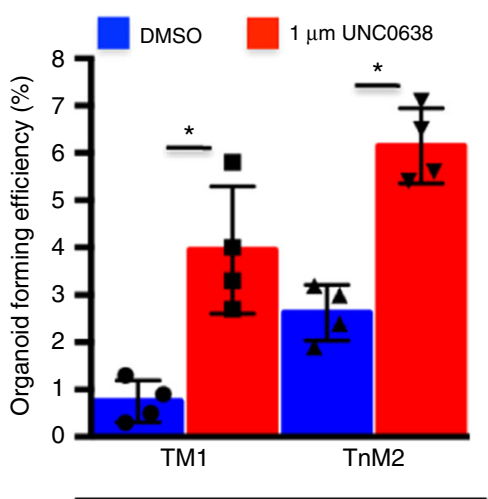

Lung adenocarcinoma cell line b

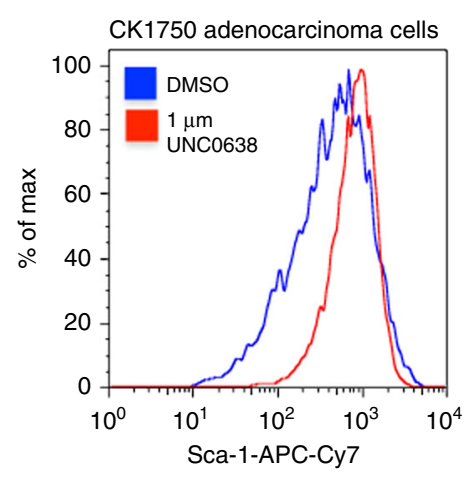

e
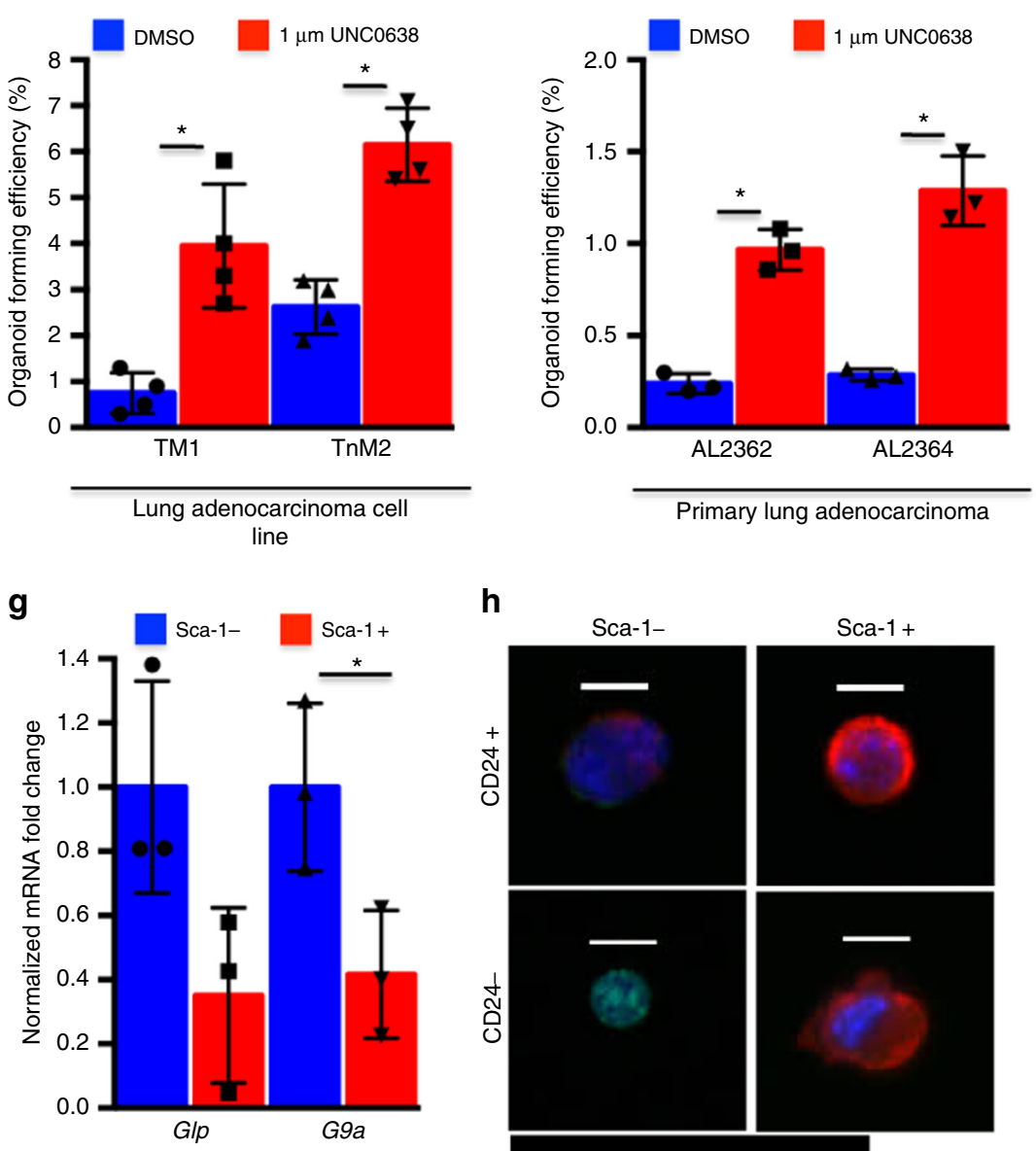

h

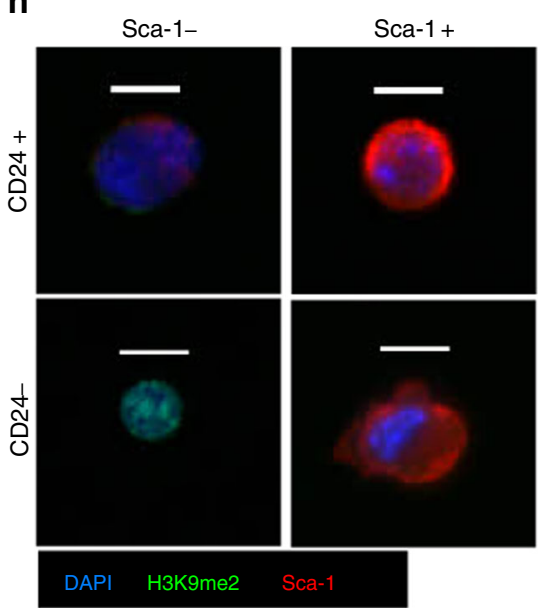

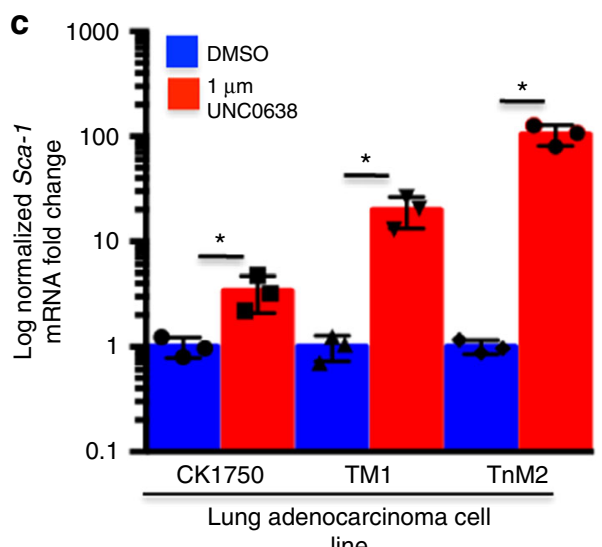

line

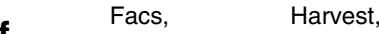

f Dose inject analyze
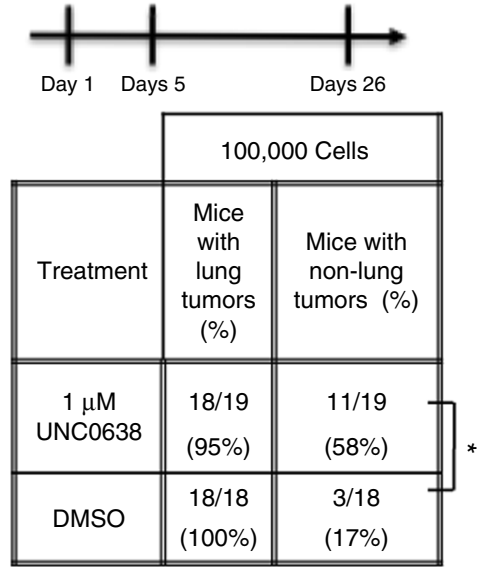

i

Sca-1- CD24-

Sca-1- CD24 +

Sca-1 + CD24-

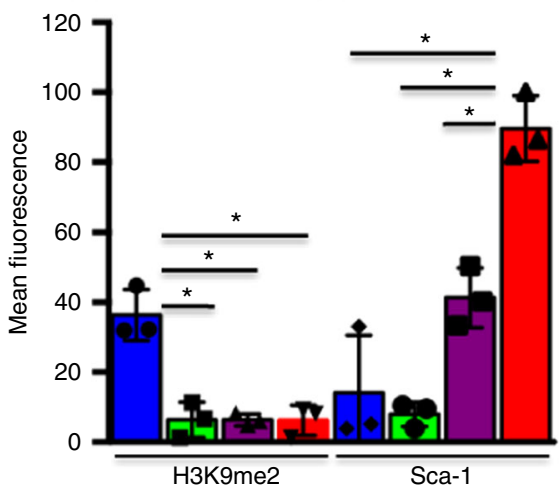

Further analysis of shG9a Kras;p53 mice supported our prediction that $\operatorname{sh} G 9 a$ would correlate with more metastasis, as we previously found that $\mathrm{CD} 24+\mathrm{Sca}-1+$ cells give rise to significantly more metastases after orthotopic transplantation compared to other tumor cell populations ${ }^{2}$. Indeed, metastases were observed in the thoracic pleura and lymph nodes in a significantly higher proportion of $s h G 9 a$ Kras;p53 primary mice compared to controls (50 vs. $0 \%, P=0.0003$ and 25 vs. $0 \%, P=$ 0.0265 , Fisher's exact test, Fig. 2d). Furthermore, fluorescenceactivated cell sorting (FACS) of metastases from shG9a Kras;p53 mice or Kras;p53 mice with tumors initiated with Adeno-Cre revealed that they almost entirely consisted of $\mathrm{CD} 24+\mathrm{Sca}-1+$
TPCs (Supplementary Fig. 4c-e). Together, the data suggest that G9a depletion can drive lung adenocarcinoma progression and promote metastasis.

G9a depletion expands the TPC population in vivo. FACS analysis of dissociated tumors revealed differences in the subpopulations of $\operatorname{sh} G 9 a$ and control tumors. We found a 1.8-fold significant increase in the CD24+Sca-1+ cell population, previously shown to be enriched in TPCs ${ }^{2}$, in $s h G 9 a$ tumors (27.7 vs. $15.6 \%, P=0.0185$, Mann-Whitney test) and a corresponding decrease in the proportion of CD24-Sca-1- cells (28 vs. 18.2\%) 
Fig. $1 \mathrm{G} 9$ a/Glp inhibition drives cells towards the TPC phenotype. a Scatter plot of fold change in median fluorescence of drug-screened CK1750 cells labeled with fluorescent anti-CD24 and anti-Sca-1 antibodies. Each point represents an individual compound, red $=$ UNC0638, blue $=$ other compounds. b Representative $(n=5)$ FACS histogram of Sca-1 fluorescence of lung adenocarcinoma cells following $96 \mathrm{~h}$. treatment with $1 \mu \mathrm{M}$ UNC0638 or vehicle control, gated for live single cells. c qPCR of Sca-1 mRNA normalized to Gapdh from adenocarcinoma cells following 96 h. treatment with $1 \mu \mathrm{M}$ UNC0638 or vehicle control. Error bars denote standard deviation. ${ }^{\star} P<0.05, t$ test, $n=3$. d Bar chart of \% of cells forming organoids 10 days after 1000 adenocarcinoma cells were plated in 3D culture with $1 \mu \mathrm{M}$ UNC0638 or DMSO following $96 \mathrm{~h}$. pre-treatment with UNC0638 or DMSO control. Error bars denote standard deviation. ${ }^{\star} P<0.05, t$ test, $n=3$. e Representative bar chart of \% of cells forming organoids 10 days after 5000 single cells isolated from a primary adenocarcinoma were plated in 3D culture with $1 \mu \mathrm{M}$ UNC0638 or DMSO. Error bars denote standard deviation. ${ }^{\star} P<0.05, t$ test, $n=3$ technical replicates. $\mathbf{f}$ Experimental design of G9ai in intravenous transplantation assay, and table of tumor incidence in recipients of UNC0638-treated or DMSOtreated adenocarcinoma cells by intravenous injection. ${ }^{\star} P<0.05$, Fisher's exact test. $\mathbf{g}$ Bar chart of mRNA abundance of G9a and Glp normalized to $\beta$-Actin in Sca-1+ cells (TPCs) relative to Sca-1- cells (non-TPCs) from FACS-sorted primary adenocarcinomas, gated for single, live, CD31-, CD45- cells. Error bars denote standard deviation. ${ }^{\star} P<0.05, t$ test, $n=3$. $\mathbf{h}$ Representative $(n=3)$ images of isolated lung adenocarcinoma cells sorted by CD24 and Sca- 1 , gated for live, single, CD31-, CD45-, Epcam+ cells after cytospin and immunostained for the indicated markers. Scale bar $=20 \mu \mathrm{m}$. $\mathbf{i}$ Quantification of H3K9me2 and Sca-1 immunostaining of isolated lung adenocarcinoma cells sorted by CD24 and Sca-1, gated for live, single CD31-, CD45-, Epcam+ cells. Error bars denote standard deviation. ${ }^{\star} P<0.05, t$ test, $n=3$

(Fig. 2e, f), reinforcing our hypothesis of G9a as a negative regulator of lung TPCs. It has also been previously reported that Notch3 is enriched in lung TPCs and is required for lung TPC function $^{3}$. In line with these findings, we observed a significant increase in tumors with nuclear Notch3 localization in $\operatorname{sh} G 9 a$ vs. $s h L u c$ recipients and in mice receiving high titer Cre-Adenovirus, which display more grade 3 and grade 4 tumors than shLuc lentivirus controls (49 vs. $10 \%, P=0.001,26 \%, P=0.008$, Mann-Whitney test) (Supplementary Fig. 4f, g).

In orthotopic transplantation experiments, recipients of $\operatorname{sh} G 9 a$ cells more frequently developed lung tumors and metastases (71 vs. $9 \%, P=0.0095,43$ vs. $0 \%, P=0.043$, Fisher's exact test) compared to mice that received control Kras;p53 cells, further confirming that $\operatorname{sh} G 9 a$ tumors have enriched TPC (Fig. 2g). To show that G9a could directly increase the frequency of TPCs, we used G9ai on wild-type lung adenocarcinoma cells and performed limiting dilution transplantation assays. G9ai cells initiated more tumors at higher dilutions than controls, increasing the estimated TPC frequency ( $1 / 14$ vs. $1 / 53, P=0.0312, \chi^{2}$ test) (Fig. $\left.2 \mathrm{~h}\right)$. These results suggest that G9a loss drives lung adenocarcinoma progression and metastasis by increasing the proportion of TPCs within the tumor.

G9a controls TPCs through KRAS-associated and ECMassociated genes. To understand how G9 controls TPC phenotypes, we sought to identify genes that acutely respond to changes in G9a by using G9ai and H3K9me2 chromatin immunoprecipitation-sequencing (ChIP-seq) and RNA-sequencing (RNA-seq). As has been observed with other cells, we found that $\mathrm{H} 3 \mathrm{~K} 9 \mathrm{me}$ covered large, Mb-sized genomic domains ${ }^{22,23}$. We did not observe changes in the locations of these domains (Supplementary Fig. 5a), even though global H3K9me2 levels were significantly reduced (Supplementary Fig. 5b), suggesting that G9ai reduced H3K9me2 evenly across the genome. RNA-seq however identified many genes differentially expressed by G9ai (Supplementary Fig. 5c). ChIP-seq showed lower $\mathrm{H} 3 \mathrm{~K} 9 \mathrm{me} 2$ across the transcription starting sites (TSS) of the RNA-seq genes commonly upregulated across multiple cell lines, and higher $\mathrm{H} 3 \mathrm{~K} 9 \mathrm{me} 2$ at the commonly downregulated genes (Supplementary Fig. 5d).

Gene ontology enrichment analysis revealed that the commonly upregulated genes were most significantly enriched in signatures associated with expression of oncogenic KRAS, as well as genes in signatures associated with the ECM, cellular secretion, and the extracellular space (Fig. 3a, Supplementary Fig. 5e). Additionally, the genes enriched within these terms were amongst the individual most upregulated genes, and several genes were present in both the KRAS-associated and ECM terms
(Supplementary Fig. 5e). We narrowed our focus to the most significantly upregulated genes enriched in the KRAS-associated and ECM terms as candidates to mediate the TPC phenotype. We confirmed the significant upregulation of multiple genes upon G9ai by qPCR across multiple adenocarcinoma cell lines (Fig. 3b, Supplementary Fig. 5f). To confirm that these genes were also regulated by G9a in vivo, we stained sections of control and shG9a tumors. Matrix metalloproteases 10 and 13 (Mmp10 and Mmp13) were expressed in $\operatorname{sh} G 9 a$ metastases and in $\operatorname{sh} G 9 a$ primary tumors, but were absent in control tumors (Fig. 3c, Supplementary Fig. 6a). We also confirmed by qPCR that a subset of these genes (Anxa10, Ctse, Mmp10, Mmp13, Lcp1) were consistently upregulated in $\operatorname{sh} G 9 a$ tumor cells compared to controls (Supplementary Fig. 6b).

Of these genes, Mmp10 was the highest ranked gene enriched in both the KRAS-associated and ECM gene signatures. Interestingly, Mmp10 has previously been reported as required for lung stem cell expansion as well as lung adenocarcinoma initiation and metastasis 24,25 , making it a logical candidate to mediate the TPC phenotypes regulated by G9a. We depleted Mmp10 from cell lines derived from $s h G 9 a$ tumors with shRNA hairpins (Supplementary Fig. 6c) and compared their tumorforming efficiency with shGFP controls. Depletion of Mmp10 in the $\operatorname{sh} G 9 a$ background significantly reduced tumor burden following intravenous transplantation ( 38.3 vs. $3.9 \%, P<0.0001$, $t$ test) (Fig. 3d), implying that Mmp10 functions in the TPC phenotype. Furthermore, ChIP-PCR showed that H3K9me2 was significantly depleted by G9ai at the TSSs of Mmp10 as well as Sca-1 $(P=0.004, P=000.5$, $t$ test) (Fig. 3e), suggesting that transcription of these genes was directly regulated by G9a.

To confirm that G9a controlled the TPC phenotype by suppressing KRAS-associated and ECM-regulating genes, we rescued $G 9 a$ expression in SR0144 cells derived from shG9a tumors. Expression of human G9a increased global H3K9me2 compared to Cre-expressing controls (Supplementary Fig. 6d, e). G9a rescue significantly repressed G9a-regulated genes Sca-1 and Mmp10 (0.03-fold, $P=0.0003$ and 0.0008 -fold, $P<0.0001, t$ test) (Fig. 3f), lowered Sca-1 surface protein levels (Supplementary Fig. 6f), and significantly reduced Mmp enzymatic activity (0.69-fold, $P=0.0082, t$ test) (Supplementary Fig. $6 \mathrm{~g}$ ). Following intravenous transplantation, G9a-rescued cells generated a significantly lower lung tumor burden than controls $(9.4$ vs. $31.9 \%, P=0.016, t$ test) (Fig. 3g), reinforcing the conclusion that G9a directly regulates TPC tumorigenicity. As G9a represses Mmp10 and other secreted proteases, we asked if G9a could regulate how tumor cells interact with the ECM. When we cultured cells in invasion assays, where serum-starved cells must migrate through a $3 \mathrm{D}$ matrix barrier towards serum-rich media, G9a-depleted cells showed 
a

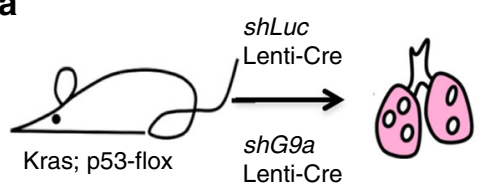

d

\begin{tabular}{|c||c||}
\hline \hline Tumor & Metastases \\
\hline \hline \multirow{2}{*}{ Control } & $0 / 19$ \\
& $(0 \%)$ \\
\hline \multirow{2}{*}{ shG9a } & $12 / 24$ \\
& $(50 \%)$ \\
\hline
\end{tabular}

b

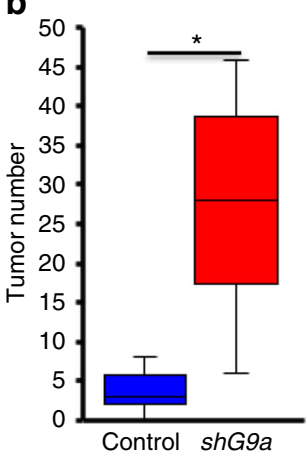

C

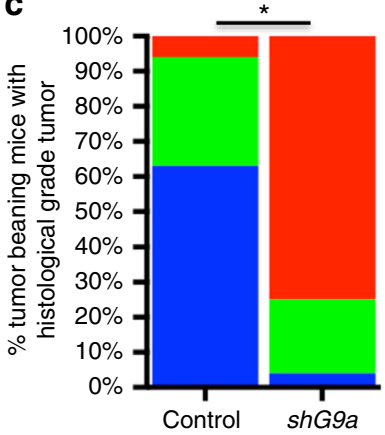

Histological grade:

Grade 2 Grade 3 Grade 4

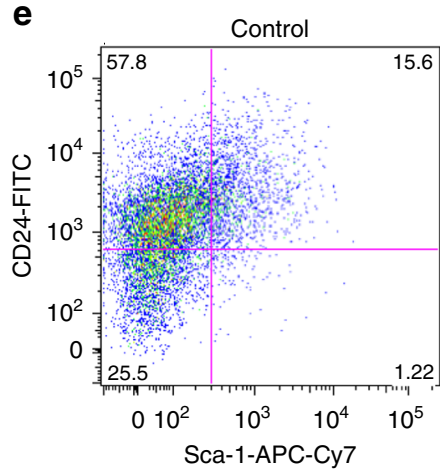

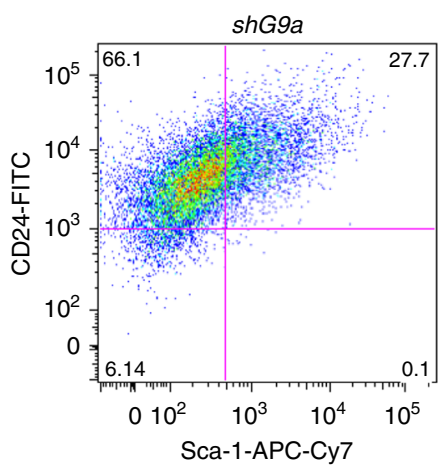

f

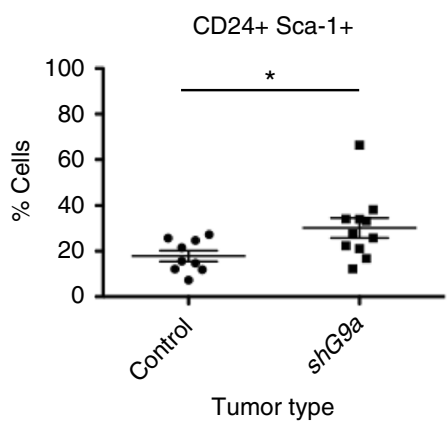

$\mathbf{h}$

\begin{tabular}{||c|c|c||c||c||c||}
\cline { 2 - 4 } \multicolumn{1}{c|}{} & \multicolumn{3}{c|}{$\begin{array}{c}\text { Number of cells } \\
\text { transplanted }\end{array}$} & \multirow{2}{\text{TPC}}{ Frequency } & $p$ Value \\
\hline \hline Cells & 1000 & 100 & 10 & & \\
\hline \hline Control & $\begin{array}{c}8 / 8 \\
(100 \%)\end{array}$ & $\begin{array}{c}6 / 8 \\
(75 \%)\end{array}$ & $\begin{array}{c}3 / 8 \\
(37.5 \%)\end{array}$ & $1 / 53$ & 0.0312 \\
\hline G9ai & $\begin{array}{c}8 / 8 \\
(100 \%)\end{array}$ & $\begin{array}{c}8 / 8 \\
(100 \%)\end{array}$ & $\begin{array}{c}4 / 8 \\
(50 \%)\end{array}$ & $1 / 14$ & \\
\hline
\end{tabular}

Fig. 2 G9a depletion expands the TPC population and promotes tumor progression and metastasis. a Cartoon of experimental design to deplete G9a in Cre-induced adenocarcinomas. $\mathbf{b}$ Box and Whisker plot of tumor number per mouse, in recipients of control and shG9a lentiviruses. Boxes represent inner quartiles, center line $=$ median, whiskers $=1.5 \times I Q R$. ${ }^{\star} P<0.05$, Mann-Whitney test. $\mathbf{c}$ Bar chart of tumor grade/mouse of control and shG9a recipients. ${ }^{\star} P<0.05, \chi^{2}$ test, $n=16$ and $n=24$ mice, respectively. d Table of metastasis occurrence in the recipients of control and shG9a lentiviruses. ${ }^{\star} P<0.05$, Fisher's exact test. e Representative FACS plots of dissociated tumors from control $(n=9)$ and shG9a $(n=11)$ recipients, gated for live, single, CD31-, CD45-, Epcam + cells. $\mathbf{f}$ Chart showing the median proportion of CD24+Sca-1+ TPCs in control and shG9a tumors determined by FACS. Each data point represents tumors from an individual mouse. ${ }^{\star} P<0.05$, Mann-Whitney test. $\mathbf{g}$ Table of secondary tumor and metastasis incidence and location in recipients of orthotopically transplanted control and shG9a tumor cells. ${ }^{\star} P<0.05$, Fisher's exact test. $\mathbf{h}$ Table of tumor incidence and TPC frequency in recipients of control or G9ai adenocarcinoma cells in limiting dilution assay. TPC frequency and $P$ value $\left(\chi^{2}\right)$ were calculated by ELDA software

significantly more invasion than controls expressing G9a $(0.69$ fold, $P=0.0002, t$ test) (Fig. 3 h). There was no difference between cells in a matrix-free migration assay (Supplementary Fig. 6h), suggesting that G9a activity more specifically regulates genes that impact the ability of cells to manipulate the matrix. In total, these data support the conclusion that one way by which G9a suppresses TPC activity is by repression of Mmp10, which in turn influences the ability to degrade the ECM.

Antagonizing H3K9 KDMs represses TPC phenotypes. G9a/Glp are not commonly mutated in lung adenocarcinoma ${ }^{26}$; therefore, we analyzed $G 9 a$ and Glp expression in the Directors Challenge data set from over 400 adenocarcinoma patients to determine if G9a may be linked to clinical criteria in patients. Glp expression was not predictive of outcome (Supplementary Fig. 7a), yet higher G9a expression was correlated with significantly better 10 -year survival ( $P=0.0075$, Mantel-Cox log-rank test) (Fig. 4a), as we would predict if G9a functioned as we have shown in the mouse. Interestingly, this contrasts with some previous studies, which have suggested that $G 9 a$ overexpression is associated with worse clinical outcome $e^{9,27}$. As chromatin modifications are bidirectionally regulated, we looked to see if any $\mathrm{H} 3 \mathrm{~K} 9 \mathrm{me} 1 / 2$ KDMs were also predictive of clinical outcome. We found one H3K9 KDM, KDM3A (also JMJD1A, JHDM2A) for which high expression correlated with worse 10 -year survival $(P=0.0022$, Mantel-Cox log-rank test) (Fig. 4b), consistent with our hypothesis that lower $\mathrm{H} 3 \mathrm{~K} 9 \mathrm{me} 1 / 2$ promotes a TPC phenotype and accelerated disease. To test if antagonizing an $\mathrm{H} 3 \mathrm{~K} 9 \mathrm{me} 1 / 2$ KDM might impair TPC phenotypes and improve outcome, we depleted $K d m 3 a$ in CK1750 mouse adenocarcinoma cells (Supplementary Fig. 7b). We observed that $\operatorname{sh} K d m 3 a$ cells displayed lower organoid-forming efficiency than control shGFP cells in 3D culture (Fig. 4c), despite their proliferation rate in adherent 
a

Top enriched gene sets in common upregulated genes
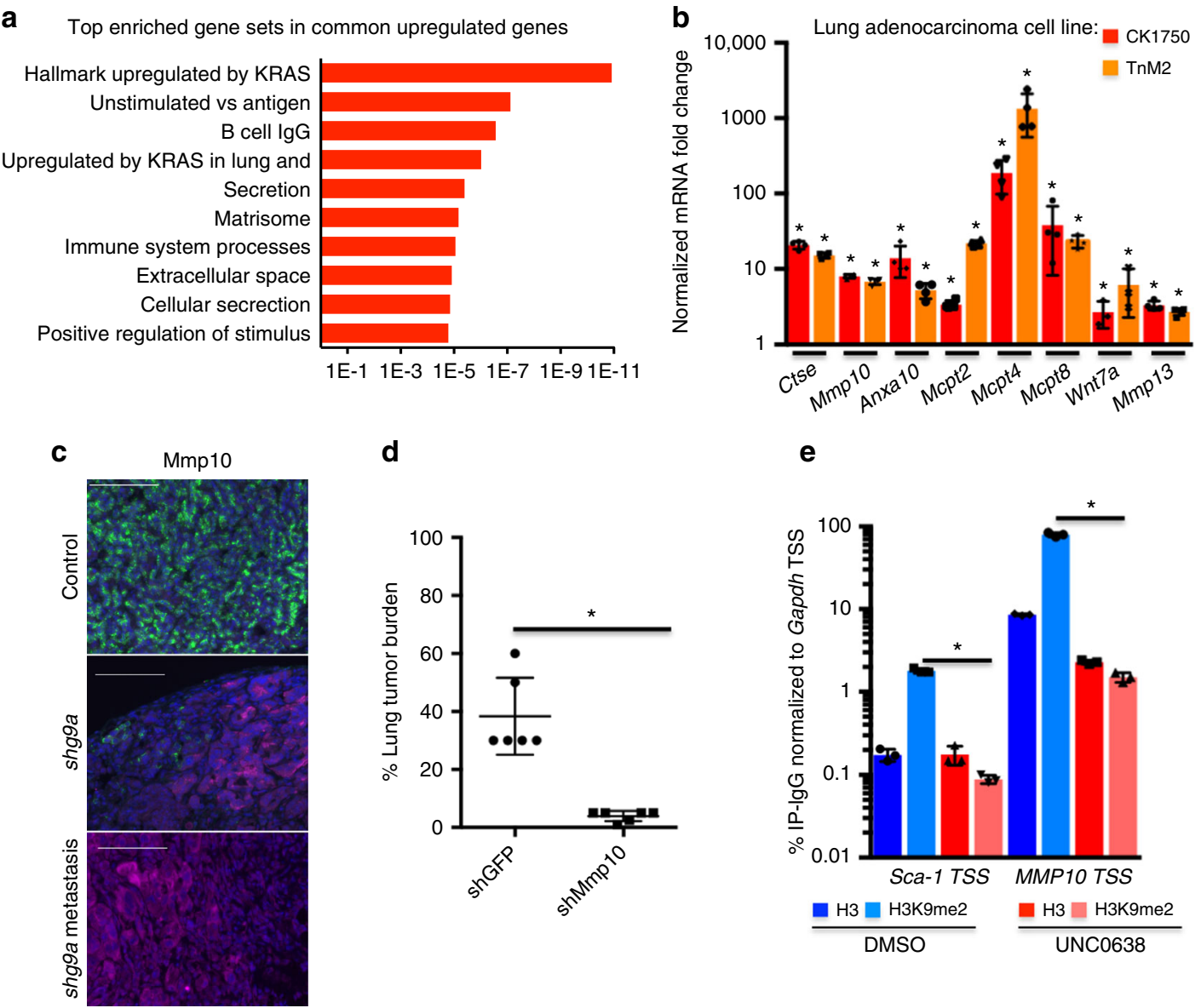

d

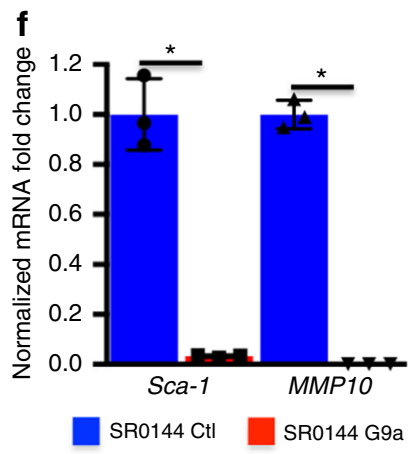

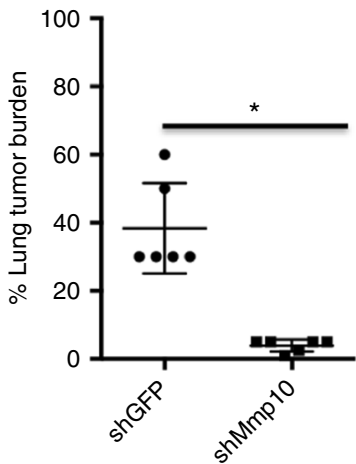

g

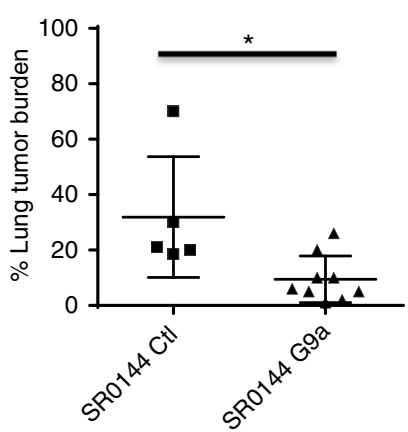

e

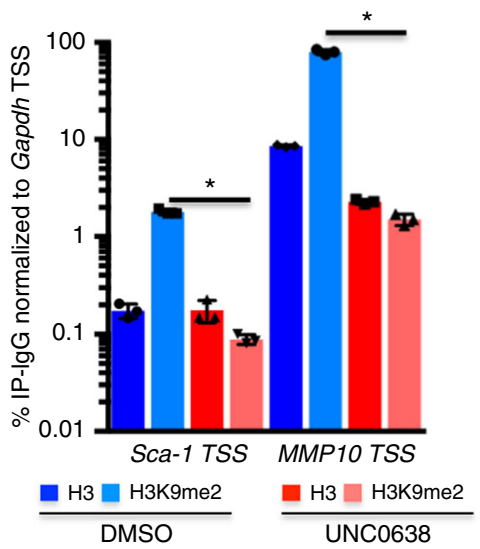

h

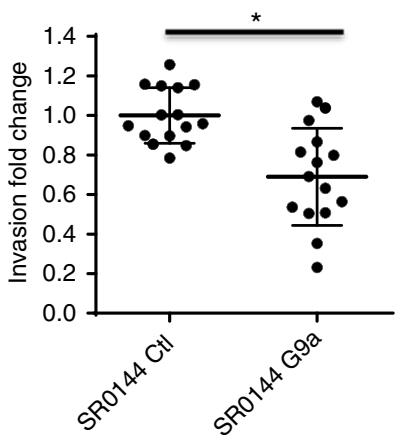

Fig. 3 G9a controls TPCs through KRAS-associated and ECM-associated genes. a Molecular Signatures Database enrichment of gene terms associated with genes upregulated upon G9a/Glp inhibition. $\mathbf{b}$ Bar chart of fold change of Gapdh-normalized mRNA levels of indicated genes in adenocarcinoma cells treated with $1 \mu \mathrm{M}$ UNC0638 relative to DMSO-treated controls. Error bars denote standard deviation. ${ }^{\star} P<0.05$, Fisher's $t$ test, $n=4$. c Representative $(n$ $=5$ ) images of shLuc control, and shG9a lung tumors and shG9a metastases immunostained for the indicated proteins. Scale bar $=100 \mu \mathrm{m}$. $\mathbf{d}$ Chart of $\%$ lung tumor area per H\&E-stained section from recipients of intravenously injected shGFP, and shMmp10 SR0144 shG9a lung adenocarcinoma cells.

${ }^{\star} P<0.05$, Fisher's $t$ test. e Representative bar chart of \% immunoprecipitation compared to input DNA-IgG of H3 and H3K9me2 ChIPs at the indicated TSS relative to Gapdh TSS of lung adenocarcinoma cells treated with $1 \mu \mathrm{M}$ UNC0638 or DMSO. Error bars denote standard deviation. ${ }^{\star} P<0.05$, Fisher's $t$ test, $n=3$ technical replicates. $\mathbf{f}$ Bar chart of mRNA from the indicated genes normalized to Gapdh from control and G9a-overexpressing SR0144 shG9a lung adenocarcinoma cells. Error bars denote standard deviation, ${ }^{\star} P<0.05$, Fisher's $t$ test, $n=3$. $\mathbf{g}$ Chart of $\%$ lung tumor area per H\&E-stained section from recipients of intravenously transplanted control and G9a-overexpressing SR0144 shG9a lung adenocarcinoma cells. ${ }^{\star} P<0.05$, Fisher's $t$ test. $\mathbf{h}$ Chart of fold change in invasion through a 3D matrix-coated migration transwell of G9a-overexpressing relative to control SR0144 shG9a lung adenocarcinoma cells. ${ }^{\star} P<0.05$, Fisher's $t$ test

culture being comparable to controls (Supplementary Fig. 7c). When $\operatorname{shKdm} 3 a$ cells were transplanted into mice, significantly fewer lung and extra-lung tumors formed compared to control cells (Fig. 4d, e), suggesting that the TPC properties of these cells were also impaired. Similarly to rescued shG9a adenocarcinoma cells (Fig. 3f), we observed significant downregulation of the G9a targets Sca-1 and $\mathrm{Mmp10}$ (Fig. 4f), suggesting that $\mathrm{Kdm} 3 \mathrm{a}$ depletion may impede TPCs through the same mechanisms as G9a expression in lung adenocarcinoma cells. When we returned to our primary mouse adenocarcinoma data, we found that a 
a

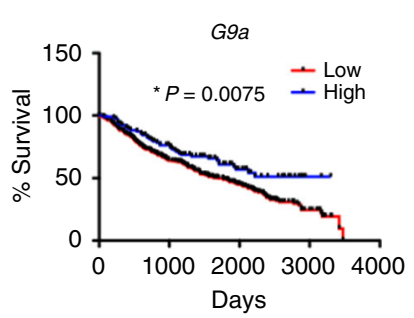

d

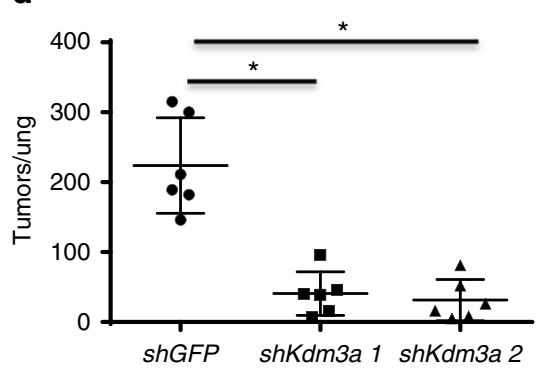

g

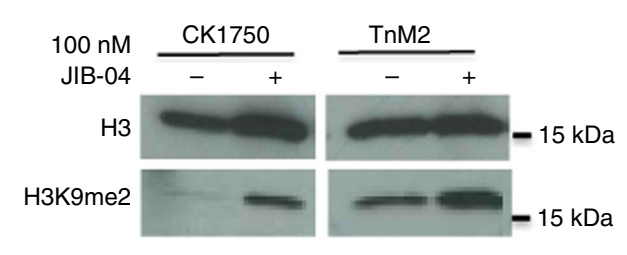

b

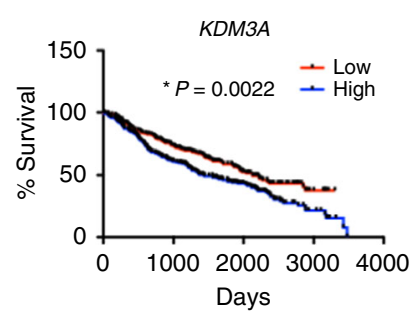

\begin{tabular}{|c|c|c|}
\hline $\begin{array}{c}500,000 \\
\text { Cells }\end{array}$ & $\begin{array}{l}\text { Mice with } \\
\text { lung } \\
\text { tumors } \\
(\%)\end{array}$ & $\begin{array}{c}\text { Mice with } \\
\text { non-lung } \\
\text { tumors } \\
(\%)\end{array}$ \\
\hline shGFP & $\begin{array}{c}6 / 6 \\
(100 \%)\end{array}$ & $\begin{array}{l}5 / 6 \\
(83 \%)]_{*}\end{array}$ \\
\hline shKdm3a 1 & $\begin{array}{c}6 / 6 \\
(100 \%)\end{array}$ & $\begin{array}{l}0 / 6 \\
(0 \%)\end{array}$ \\
\hline "shKdm3a 2 & $\begin{array}{c}6 / 6 \\
(100 \%)\end{array}$ & $\begin{array}{l}0 / 6 \\
(0 \%)\end{array}$ \\
\hline
\end{tabular}

$\mathbf{f}$

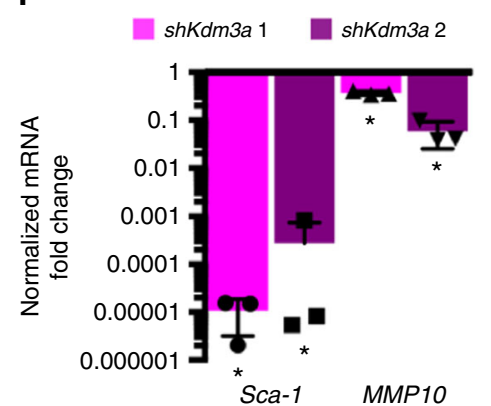

h Mouse lung adenocarcinoma (TM1)
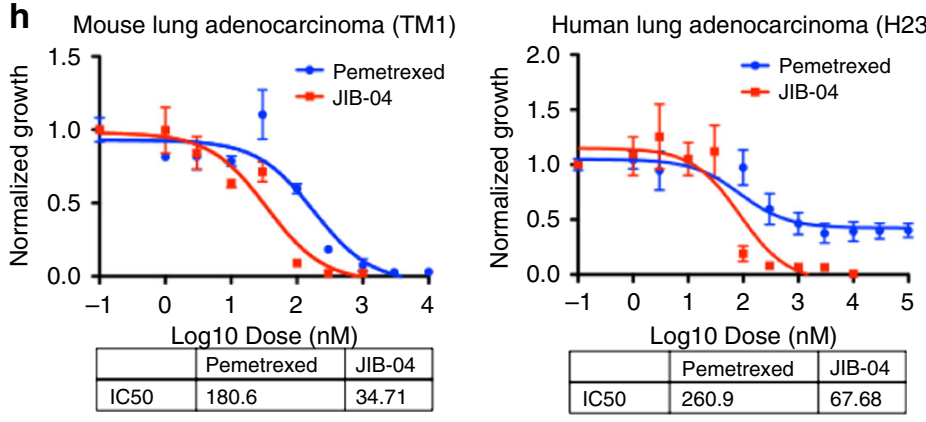

$\mathbf{i}$

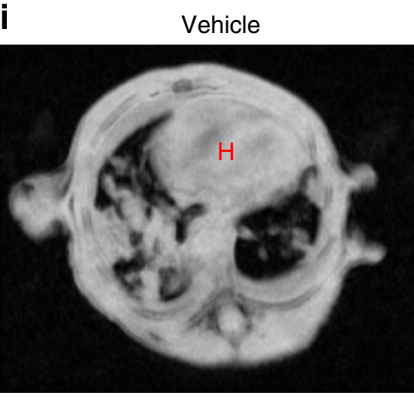

JIB-04

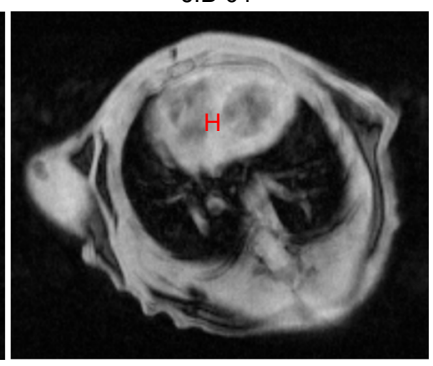

j

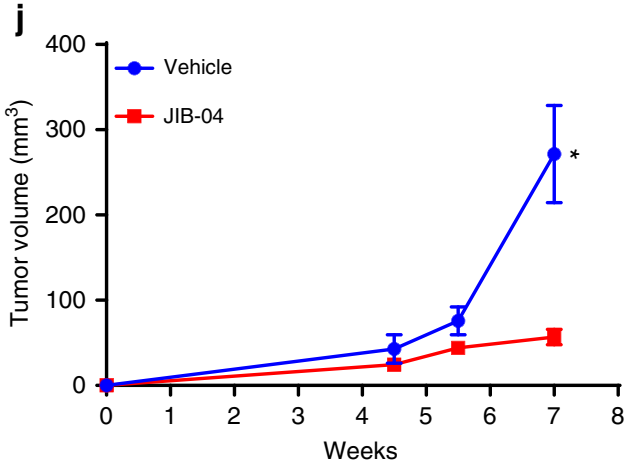

Fig. 4 Antagonizing H3K9 KDMs represses TPC phenotypes. a, b Kaplan-Meier curves of 10-year survival of patients from the director's challenge cohort of early stage lung adenocarcinoma. Populations sorted for high and low expression of a H3K9me1/2 G9a and b H3K9me1/2 demethylase KDM3A. *P< 0.05 , Mantel-Cox log-rank test. c Bar chart of \% of cells forming organoids 10 days after 1000 shGFP and shKdm3a CK1750 adenocarcinoma cells were plated in 3D culture. Error bars denote standard deviation. ${ }^{\star} P<0.05$, Fisher's $t$ test, $n=4$. d Chart of average number of tumors per H\&E-stained section from recipients of intravenously transplanted shGFP and shKdm3a adenocarcinoma cells. ${ }^{\star} P<0.05$, Mann-Whitney test. e Table of transplanted mice with lung and non-lung tumors. ${ }^{*} P<0.05$, Fisher's exact test. $\mathbf{f}$ Bar chart of fold change of Gapdh-normalized mRNA levels of indicated genes in shKdm3a adenocarcinoma cells over shGFP control cells. Error bars denote standard deviation. ${ }^{\star} P<0.05$, Fisher's $t$ test, $n=3$. $\mathbf{g}$ Western blot of whole cell lysates from CK1750 adenocarcinoma cells treated with either $100 \mathrm{nM} \mathrm{JIB-04}$ or DMSO for $96 \mathrm{~h}$, and then immunoblotted for the indicated antibodies. $\mathbf{h}$ Representative dose-response curve of mouse (TM1) and human (H23) NSCLC cells grown for $96 \mathrm{~h}$ with JIB-04 or Pemetrexed, normalized to growth with vehicle control. i Representative MR images of allograft mice treated with vehicle or JIB- 04 at 7 weeks post transplantation. $\mathrm{H}$ indicates heart area. $\mathbf{j}$ Chart of average tumor volume of allografted mice treated with either vehicle or JIB-04. ${ }^{\star} P<0.05$, Fisher's $t$ test 

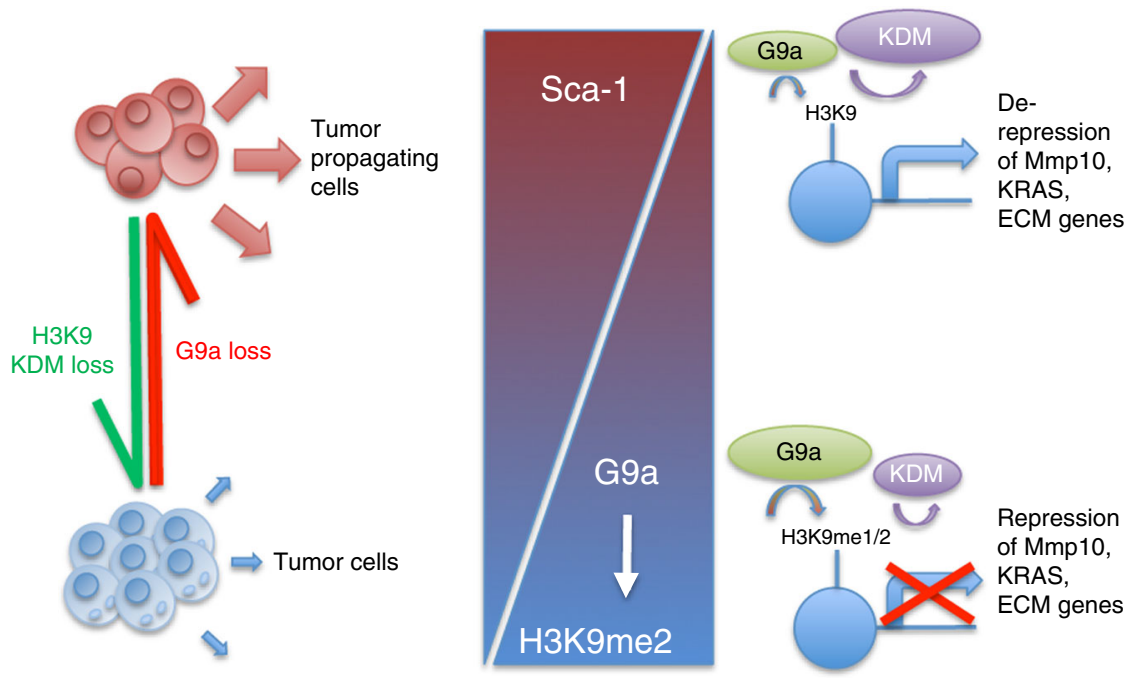

Fig. 5 H3K9 methyltransferases and demethylases control lung tumor-propagating cells through transcriptional regulation of Mmp10, KRAS and ECM genes. In lung adenocarcinoma non-stem tumor cells, G9a is relatively highly expressed and H3K9me2 KDMs are relatively under-expressed. Chromatin is relatively enriched in $\mathrm{H} 3 \mathrm{~K} 9 \mathrm{me2}$, which represses the transcription of Sca-1, Mmp10, and other genes associated with mutant KRAS and ECM regulation. Loss of G9a or gain of H3K9me2 KDMs reduces H3K9me2 and allows for the expression of previously repressed genes. This enables tumor cells to acquire the tumor-propagating cell phenotype, and the expansion of the tumor-propagating cell population drives lung adenocarcinoma progression and metastasis. Re-expression of G9a or antagonism of H3K9me2 KDMs can reverse the TPC phenotypes, mitigating lung adenocarcinoma progression

different $\mathrm{H} 3 \mathrm{~K} 9 \mathrm{me} 1 / 2$ demethylase, Phf8, was significantly upregulated in Sca-1+ TPCs (3.6-fold, $P=0.022, t$ test) (Supplementary Fig. 7d). We also depleted Phf8 in CK1750 cells (Supplementary Fig. 7e, f), and found that similarly shPhf8 cells had a lower organoid-forming efficiency (Supplementary Fig. 7g), formed fewer tumors when transplanted (Supplementary Fig. 7h), and had reduced Sca-1 and Mmp10 expression (Supplementary Fig. 7i).

Currently, there are no inhibitors that specifically target Kdm3a or Phf8; however, there are several broad-spectrum KDM inhibitors, which will inhibit these enzymes along with other KDMs. We found that pan-jumonji inhibitor JIB- $04^{28}$ increased $\mathrm{H} 3 \mathrm{~K} 9 \mathrm{me} 2$ levels (Fig. 4g), and also resulted in the loss of surface Sca-1 in adenocarcinoma cells (Supplementary Fig. 7j). Furthermore, JIB-04 affected the viability of both mouse and human adenocarcinoma and squamous non-small-cell lung cancer (NSCLC) cell lines. JIB-04 had an approximately equal or lower half-maximal inhibitory concentration $\left(\mathrm{IC}_{50}\right)$ in both mouse and human cell lines than Pemetrexed, a current frontline therapeutic for the treatment of NSCLC (34.71 vs $180.6 \mathrm{nM}, 67.68$ vs $260.9 \mathrm{nM}$, Fig. $4 \mathrm{~h}$ ). We also performed allograft transplantations of adenocarcinoma cells into immunologically competent host mice and treated them with either vehicle or JIB-04. After 7 weeks, relative tumor volume was significantly higher in the vehicle cohort compared to JIB-04-treated mice (271.35 vs. 56.7 $\mathrm{mm}^{3}, P=0.0014, t$ test) (Fig. $4 \mathrm{i}, \mathrm{j}$ ), suggesting that targeting TPCs through H3K9me KDM inhibition could be an effective therapy for lung adenocarcinoma.

\section{Discussion}

We found that inhibition of G9a, an HMT previously reported to have oncogenic activity, leads to enhanced TPC function and drives metastatic tumor progression. In vitro and in vivo studies revealed that G9a represses a gene signature highly associated with mutant KRAS-regulating and ECM-regulating genes, including Mmp10, which partially mediates the tumorigenicity of G9a-depleted tumor cells. Conversely, we found that inhibition of H3K9 KDMs (KDMi) may be a beneficial treatment for advanced lung adenocarcinoma. By studying the epigenetic dependencies of
TPCs that represent a minority of tumor cells, our work has revealed a potential new therapeutic intervention for advanced lung cancer (Fig. 5).

Since the discovery of potent G9a/Glp inhibitors, there has been an increase in studies investigating these enzymes in cancer. Indeed, much of the rationale for developing G9a/Glp inhibitors was that many tumors stained positively for these enzymes and this was often correlated with worse outcome ${ }^{14,15,29}$. It has been observed amongst almost all cancer models tested that G9a inhibition has antiproliferative effects, and different mechanisms have been proposed ${ }^{9,10}$. While we found that high concentrations of G9a inhibitors were antiproliferative, we also observed that G9a is downregulated in highly tumorigenic TPCs (and in an H3K9 KDM, Phf8 is upregulated), coinciding with a significant reduction in global H3K9me2. Our data suggest that downregulation of G9a promotes TPCs, leading to disease progression, metastasis, and worse clinical outcome, thus raising caution for the use of G9a inhibitors, particularly for lung cancer treatment.

Our studies suggest that TPCs can promote tumorigenesis and metastasis by influencing the extracellular environment. While our data are consistent with previous studies which found that deleting or inhibiting G9a can increase stem cell or tumor stemlike populations and oppose tissue-specific differentiation ${ }^{23,30}$, we focused this study on the role of G9a in regulation of genes affecting ECM. Mmp10, the G9a target we characterized in lung cancer cells, directly mediates TPC tumorigenicity. In addition to degrading the ECM to facilitate invasion, Mmp10 has also been proposed to promote metastasis by proteolytically regulating extracellular pools of growth factors such as TGF- $\beta$ and

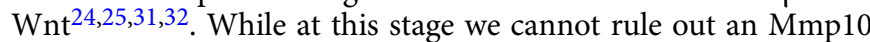
effect on these signaling pathways, we favor the hypothesis that Mmp10 expressed by lung TPCs acts on the ECM, as we observed that G9a-overexpressing cells repress Mmp10, have lower Mmp enzymatic activity, and are partially impaired in invading through a $3 \mathrm{D}$ matrix. It has been hypothesized that the tumor microenvironment influences TPCs, but the ability of TPCs to alter their surroundings via ECM remodeling warrants further study.

Mmps are strongly associated with poor survival in lung cancer, but to date trials of Mmp inhibitors have not proven successful, 
through the lack of efficacy, toxicity, or poor bioavailability ${ }^{33}$. We found that Mmp10 is de-repressed by $\mathrm{H} 3 \mathrm{~K} 9 \mathrm{KDMs}$; therefore, KDMi may prevent or delay the re-expression of these genes, potentially providing another angle to target Mmps in lung cancer. Other studies have also found that KDMs are upregulated in cancers and this correlates with worse outcome $\mathrm{e}^{28,34}$. This further suggests that KDMi could be a useful therapeutic intervention, particularly as chemoresistance is a proposed TPC trait ${ }^{35}$. JIB-04 is a nonspecific KDM inhibitor and it is unlikely that all the effects we observe are due to its inhibition of H3K9 KDMs. While several more specific KDM inhibitors exist ${ }^{36,37}$, there are none that inhibit all the H3K9me KDMs. The development of pan H3K9me KDM inhibitors would be of great interest and potentially therapeutic benefit for highly refractive diseases such as lung adenocarcinoma. As KRAS mutations are common, but KRAS itself is not a druggable target, our finding that $\mathrm{H} 3 \mathrm{~K} 9 \mathrm{KDMi}$ decreases expression of genes associated with oncogenic KRAS suggests that $\mathrm{H} 3 \mathrm{~K} 9 \mathrm{KDMi}$ represents a viable personalized therapy for patients with KRASmutated adenocarcinoma.

This study has highlighted the importance of choosing the best model of cellular heterogeneity for analyses that may lead to new therapeutic approaches in cancer. Studies primarily based on cell lines or whole tumors have suggested that G9a is oncogenic and a good target for epigenetic therapy, but our approach, beginning with analyzing different tumor populations, led to a startlingly different conclusion. We propose that analyzing the effects of new therapeutics on different tumor populations, especially the most tumorigenic stem-like cells, would be greatly beneficial. This may increase the success rate of clinical trials and thus reduce the cost and time taken to bring new cancer therapeutics to the patient.

\section{Methods}

Cell lines. Mouse cell lines CK1750, SC241, and SR0144 were generated as previously described ${ }^{2}$. Cell lines TM1 and TnM2 were previously provided by Monte Winslow. Mouse cell lines were cultured in Dulbecco's modified Eagle's medium (DMEM) $+10 \%$ fetal bovine serum (FBS), 4mM L-glutamine, and penicillin/ streptomycin at $37{ }^{\circ} \mathrm{C}$ with $5 \% \mathrm{CO}_{2}$. Human $\mathrm{H} 23$ cells (ATCC CRL-5800) were used as previously described ${ }^{38}$, cultured in RPMI-1640 media with $10 \% \mathrm{FBS}, 4 \mathrm{mM}$ L-glutamine, and $50 \mathrm{U} / \mathrm{ml}$ penicillin/streptomycin at $37^{\circ} \mathrm{C}$ with $5 \% \mathrm{CO}_{2}$.

Vectors. The pLKO.1 Mmp10, Kdm3a and Phf8 shRNA construct clones TRCN000031240, TRCN000031241, TRCN0000252743, TRCN0000252745, TRCN0000086826and TRCN0000086827 were purchased from Sigma and the shGFP plasmid 12273 was available from Addgene ${ }^{38}$. The $G 9 a$ expression vector ${ }^{39}$ 41721 and Cre expression control vector ${ }^{40} 34565$ were also purchased from Addgene. The shRNA EHMT2 (G9A) lentiviral vectors were created by cloning hairpin oligonucleotides into the U6shRNA pgkCre backbone, created by the Tyler Jacks lab and available from Addgene as previously described ${ }^{41}$. The vectors used were as follows: shLuciferase-FWD, 5'-5Phos/TGA GCT GTT TCT GAG GAG CCT TCA AGA GAG GCT CCT CAG AAA CAG CTC TTT TTT C-3' and REV, 5'-5Phos/CGA GAA AAA AGA GCT GTT TCT GAG GAG CCT CTC TTG AAG GCT CCT CAG AAA CAG CTC A-3'; shEHMT2 1-FWD, 5'-5Phos/TGG CTG CTC CAG GAG TTT AAT TCA AGA GAT TAA ACT CCT GGA GCA GCC TTT TTT C-3' and REV, 5'-5Phos/TCG AGA AAA AAG GCT GCT CCA GGA GTT TAA TCT CTT GAA TTA AAC TCC TGG AGC AGC CA-3'; shEHMT2 2 FWD, 5'-5Phos/TGG AAG AGG AGG AAG AAG AAT TCA AGA GAT TCT TCT TCC TCC TCT TCC TTT TTT C- $3^{\prime}$ and REV, $5^{\prime}$-5Phos/TCG AGA AAA AAG GAA GAG GAG GAA GAA GAA TCT CTT GAA TTC TTC TTC CTC CTC TTC CA-3'.

Generation, concentration, and infection of mice with lentivirus was performed as previously described ${ }^{42}$.

Chemical compounds. The Stem Cell Chemical Library was assembled from commercially available compounds, and diluted to a stock of $10 \mathrm{mM}$ in DMSO. Chemicals were further diluted to $20 \mu \mathrm{M}$ in DMEM before being added to the cells. The chemicals used are listed in Table 1. UNC0638 was purchased from Cayman Chemical and diluted to a stock of $10 \mathrm{mM}$ in DMSO for all cell culture experiments. JIB-04 was purchased from Tocris was diluted to a stock of $10 \mathrm{mM}$ in DMSO for cell culture experiments and $5.5 \mathrm{mg} / \mathrm{ml}$ in Koliphor-EL (Sigma) for mouse experiments. Pemetrexed was purchased from the Dana Faber Cancer Center and was diluted to a stock of $100 \mathrm{mM}$ in $\mathrm{dd}_{2} \mathrm{O}$. For cell culture experiments, cells were grown in the presence of the drug for $96 \mathrm{~h}$ with fresh media and drugs were supplied at $48 \mathrm{~h}$.

Flow cytometry of in vitro cultured cells. For flow cytometry screening, CK1750 cells were plated at 300 cells/well in 96-well plates and treated with compounds from the Chemical Library for $96 \mathrm{~h}$. For routine flow cytometry analysis, cells were grown and cultured as described above. Cells were then dissociated, washed, and re-suspended in $2 \%$ FBS in phosphate-buffered saline (PBS) (PF2) and stained with anti-CD24-PE (BD Pharmingen 553262, 1:100), anti-Sca-1-APC-Cy7 (BD Pharmingen $560654,1: 100)$, and DAPI (4',6-diamidino-2-phenylindole) for $15 \mathrm{~min}$ on ice. Cells were washed again and re-suspended in $50 \mathrm{mM}$ EDTA, 2\% FBS in PBS, filtered $(40 \mu \mathrm{M})$, and sorted on a BD LSR II flow cytometer. Data were analyzed with FACSDIA (BD) and FlowJo software (Tree Star). For general flow cytometry analysis of cell surface markers, cells were cultured as described and stained as above and sorted on a BD Fortessa. Example gating strategy is illustrated in Supplementary Figure 8.

Mouse FACS analysis and sorting. Mice were euthanized with avertin overdose and lungs were dissected and examined grossly for tumor formation. Tumors were dissected from the lungs of primary mice and tumor tissue was prepared as described $^{2}$. Briefly, tumors were isolated, minced, digested rotating for $1 \mathrm{~h}$ at $37^{\circ} \mathrm{C}$ with $2 \mathrm{mg} / \mathrm{ml}$ collagenase/dispase (Roche), and then filtered twice $(100 \mu \mathrm{m}$, and then $40 \mu \mathrm{m}$ ) after a $5 \mathrm{~min}$ incubation with $0.025 \mathrm{mg} / \mathrm{ml}$ DNase. Cell lines were trypsinized and then filtered $(40 \mu \mathrm{m})$. Single-cell suspensions were stained using antiCD31-APC (BD Pharmingen 551262), anti-CD45-APC (BD Pharmingen 559864), anti-Epcam-PE-Cy7 (BioLegend 118216), anti-CD24-FITC (BD Pharmingen 553261), anti-Sca-1-APC-Cy7 (BD Pharmingen 560654), with DAPI (Sigma) staining to visualize dead cells. All antibodies were incubated for 15-20 min at 1:100 dilutions. Cell sorting was performed with a Beckman Coulter/Cytomation MoFlo or a BD FACS Aria, and data were analyzed with the FlowJo software (Tree Star Inc.). Example gating strategy is illustrated in Supplementary Figure 9.

Quantitative RT-PCR. qPCR was carried out as previously described ${ }^{2}$. Cultured cells or tissues were harvested and dissociated according to their specific protocols. RNA was isolated using the Absolutely RNA Microprep Kit (Agilent). Complementary DNA was made using the SuperScript III Kit (Invitrogen) and analyzed using TaqMan Assays (Applied Biosystems) with a StepOnePlus ${ }^{\text {ot }}$ Real-Time PCR System (Applied Biosystems) and software as per the manufacturer's recommendations. Gapdh (4352339E) was used as an endogenous control for normalization.

Organoid-forming assays. For organoids derived from cultured adenocarcinoma cells or primary tumors, dissociated single cells were re-suspended in a 1:1 ratio of GFR Matrigel (Corning) and DMEM/F12 supplemented with $1 \times$ ITS (Corning) and $95 \mu \mathrm{l}$ of cell mixture was plated in $6.5 \mathrm{~mm}, 0.4 \mu \mathrm{M}$ membrane transwell plates (CoStar). Either 1000 (cell lines) or 5000 cells (Primary cells) were plated per well. Four hundred and ten miroliters of DMEM/F12 was plated beneath the transwell and was refreshed every $48 \mathrm{~h}$. At the end of the culture period, organoids were counted.

Tumor transplant assays. Intratracheal transplants of primary sorted tumor cells were performed as described ${ }^{1,2}$. Mice were monitored for signs of lung tumor onset and euthanized for gross and histological analysis upon signs of distress. Tail vein injections were performed as previously described ${ }^{2}$, cells were re-suspended in PBS, and mice were sacrificed 2 weeks after injection for analysis of lung metastasis. For limiting dilution assays, cells were re-suspended in PBS and diluted to the appropriate concentrations. Cell suspensions were then mixed 1:1 to Matrigel and injected subcutaneously into immunocompromised mice as previously described ${ }^{2}$ Mice were sacrificed after 3 weeks and tumors were measured and analyzed.

Western blotting. Western blotting was performed as was previously described ${ }^{38}$ using the following antibodies and concentrations: H3K9me2 (Ab1220, Abcam, 1:1000) and histone H3 (ab1791, Abcam, 1:10,000). Uncropped scans are displayed in Supplementary Figure 10.

Proliferation assays. Cells were plated at 500 cells per well in 24-well plates and grown for $192 \mathrm{~h}$, with media changes every $48 \mathrm{~h}$ and wells fixed at timepoints with $10 \%$ formalin. Fixed cells were stained with Crystal violet and eluted stain was measured for OD 600 absorbance on a Synergy Neo plate reader.

Microarray analysis. Microarray analysis was performed as previously described ${ }^{2}$.

ChIP and ChIP-seq. Treated cell lines were crosslinked with $1 \%$ formaldehyde in PBS for $10 \mathrm{~min}$ at room temperature (RT), washed in $5 \mathrm{mg} \mathrm{ml}^{-1}$ bovine serum albumin in PBS and then in just cold PBS, re-suspended in lysis buffer $(50 \mathrm{mM}$ Tris-HCl, $\mathrm{pH}$ 8.1, $10 \mathrm{mM}$ EDTA, $1 \%$ sodium dodecyl sulfate (SDS), $1 \times$ complete protease inhibitors (Roche)) and sonicated with the Covaris M220 sonicator to obtain chromatin fragment lengths of 200-500 bp judged by Bioanalyzer DNA High Sensitivity Kit (Agilent). Fragmented chromatin was diluted in 
immunoprecipitation (IP) buffer (20 mM Tris- $\mathrm{HCl}, \mathrm{pH} 8.1,150 \mathrm{mM} \mathrm{NaCl}, 2 \mathrm{mM}$ EDTA, $1 \%$ Triton X-100) and incubated overnight at $4{ }^{\circ} \mathrm{C}$ with Protein $\mathrm{G}$ magnetic beads (Dynabeads: Life Technologies) that had been pre-incubated with H3K9me2 (Abcam, ab1220, $62.5 \mathrm{ng} / \mu \mathrm{l}$ ) or H3K9me1 (Abcam, ab8896, $62.5 \mathrm{ng} / \mu \mathrm{l}$ ) antibodies. Immunoprecipitates were washed six times with wash buffer $(50 \mathrm{mM}$ HEPES, $\mathrm{pH}$ 7.6, $0.5 \mathrm{M} \mathrm{LiCl}, 1 \mathrm{mM}$ EDTA, $0.7 \% \mathrm{Na}$ deoxycholate, $1 \% \mathrm{NP}-40$ ) and twice with TE buffer. Immunoprecipitated (or no IP input) DNA was treated with RNase A and proteinase $\mathrm{K}$ on the beads, recovered in $1 \%$ SDS and $0.1 \mathrm{M} \mathrm{NaHCO}_{3}$ over a period of $5 \mathrm{~h}$ at $65^{\circ} \mathrm{C}$, and column purified with DNA clean and concentrator-25 (Zymo Research). One to ten nanograms of DNA were used for the library construction using NEBNext Ultra DNA Library Prep Kit (NEB E7370). Sequencing was performed on a NextSeq (Illumina) for 38 nucleotides from paired ends according to the manufacturer's instructions.

Sequenced reads were mapped to reference mouse genome build 38 ( $\mathrm{mm} 10)$, using bowtie2 aligner. H3K27Ac-modified regions were identified using MACS (version 1.4.2) as previously described ${ }^{43}$, with a $P$ value cutoff of $1 \mathrm{E}-5$ and with default values for other parameters. Computer code is available upon request. Quantitative differences of histone modification between treated and non-treated cell lines were analyzed using the MAnorm algorithm with a $P$ value cutoff of $1 \mathrm{E}$ -10 and $>1 \log 2$ fold change.

Wiggle files with a $10 \mathrm{bp}$ resolution for $\mathrm{H} 3 \mathrm{~K} 9 \mathrm{me} 2$ and $\mathrm{H} 3 \mathrm{~K} 27 \mathrm{ac}$ modifications were generated by MACS v1.4.2 with tag shift and then rescaled to a larger of total number of uniquely alignable sequences in two data sets. Histone modification profiles were then generated with the "heatmap" function or the SitePro module in Cistrome analysis pipeline (http://cistrome.org/). Wiggle files were also visualized in the Integrative Genomics Viewer ${ }^{44}$. ChIP-seq experiments were performed with an $n$ of four biological replicates

For ChIP-PCR, purified DNA from IPs as described using H3K9me2 (Ab1220, Abcam), histone H3 (ab1791, Abcam), or IgG antibodies and input DNA was amplified using the SYBR Green (Applied Biosystems) with a StepOnePlus ${ }^{\text {wa }}$ RealTime PCR System (Applied Biosystems) and software as per the manufacturer's recommendations. The primer sequences used were as follows: Gapdh TSS-FWD, $5^{\prime}$-CTC TCT TTG GAC CCG CCT CAT TT- $3^{\prime}$ and REV, $5^{\prime}$-GAG TCC TAT CCT GGG AAC CAT CAC-3'; Sca-1 TSS-FWD, 5'-TAA CT TTT CCA GGC CAT CAT- $3^{\prime}$ and REV, $5^{\prime}$-GTT CCT CCC CAA CTG CTA TAA- $3^{\prime}$; $M m p 10$ TSSFWD, 5'-TGA CCT AAA CAC CGA CTC CCT-3' and REV, 5' -AGG ATG GCT AGT GGC TCC AT-3'.

RNA-sequencing. RNA-seq was performed by the Molecular Biology Core Facilities at the Dana-Farber Cancer Institute. RNA libraries were prepared using Illumina TruSeq Stranded mRNA Sample Preparation Kits from $500 \mathrm{ng}$ of purified total RNA according to the manufacturer's protocol. The resultant dsDNA libraries were quantified by Qubit fluorometer, Agilent TapeStation 2200, and quantitative reverse transcription PCR using the Kapa Biosystems Library Quantification Kit according to the manufacturer's protocols. Uniquely indexed libraries were pooled in equimolar ratios and sequenced on a single Illumina NextSeq500 run with single-end 75 bp reads by the Dana-Farber Cancer Institute Molecular Biology Core Facilities.

Sequenced reads were aligned to the UCSC $\mathrm{mm} 9$ reference genome assembly and gene counts were quantified using STAR (v2.5.1b $)^{45}$. Differential gene expression testing was performed by DESeq2 (v1.10.1) ${ }^{46}$ and normalized read counts (RPKM) were calculated using cufflinks (v2.2.1 ${ }^{47}$. RNA-seq analysis was performed using the VIPER snakemake pipeline (https://bitbucket.org/cfce/viper/).

Immunofluorescence and immunohistochemistry. Immunofluorescence of tumor sections was performed as previously described ${ }^{2}$, with antibodies for proSPC (1:200, Santa Cruz), G9a (ab185050, Abcam, 1:200), Notch3 (5276S, Cell Signaling, 1:100), Mmp10 (ab59437, Abcam, 1:100), Mmp13, (ab39012, Abcam, $1: 100$ ), and Mcpt4 (ab92368, Abcam, 1:100). Immunohistochemistry was performed as described ${ }^{2}$ with anti-Ki-67 (1:10,000, Novocastra) antibodies and developed using Vectastain Elite ABC Kit (Vector Labs). Imaging was performed with a Nikon 90i camera and NIS-Elements software and processed with NISElements and Adobe Photoshop.

Mice and tissues. Lox-Stop-Lox-Kras ${ }^{G 12 D} ; p 53^{f l / f l}$ and Lox-Stop-Lox-Kras ${ }^{G 12 D}$ mice ${ }^{48,49}$ were maintained in virus-free conditions in the 129 Svjae background. All mouse experiments were approved by the $\mathrm{BCH}$ Animal Care and Use Committee and by the Dana-Farber Cancer Institute Institutional Animal Care and Use Committee, both accredited by AAALAC, and were performed in accordance with relevant institutional and national guidelines and regulations. Lung tissue preparation was as described ${ }^{2}$ and sections were analyzed for tumors by at least two investigators including a pathologist with expertise in murine lung cancer. Tumor burden, the percentage of lung filled with tumor, was calculated using ImageJ to measure total lung and tumor area from hematoxylin and eosin (H\&E)-stained paraffin sections from each mouse. For metastases, heart, liver, spleen, kidneys, lymph nodes, and chest wall were also analyzed.

Kaplan-Meier analysis. Raw gene expression data from the Director's Challenge human lung adenocarcinoma samples ${ }^{50}$ were obtained (https://caintegrator.nci.nih. gov/caintegrator/). Probe intensities from the Affymetrix U133A platform used in these studies were normalized and modeled using the dChip software ${ }^{51}$ (http:// biosun1.harvard.edu/complab/dchip). Kaplan-Meier survival analyses were implemented after the samples were hierarchically clustered based on EHMT2 (G9a), KMD3A, EHMT1 (Glp), or PHF8 expression. Survival differences between the two risk groups were assessed using the Mantel-Cox log-rank test.

Cell viability assays. Cell lines were dissociated, counted, and plated at 3000 cells per well in 96-well plates with drug concentrations of the following doses: JIB-04$0,1,3,10,30,100,300$, and $1000 \mathrm{nM}$; Pemetrexed-0, 1, 10, 100, 300, 1000, 3000, and $10,000 \mathrm{nM}$. Edge wells were filled with PBS. Fresh media and drug were added after $48 \mathrm{~h}$. After $96 \mathrm{~h}$, cells were fixed with $10 \%$ formalin. Fixed cells were stained with Crystal violet and eluted stain was measured for OD 600 absorbance on a Synergy Neo plate reader. Results from independent biological replicate experiments were input into the GraphPad Prism software to extrapolate $\mathrm{IC}_{50}$ and s.e.m. of $\mathrm{IC}_{50}$ for a given cell line using the nonlinear regression analysis of $\log$ (inhibitor) vs. normalized response with a variable slope.

Invasion and migration assays. Invasion and migration assays were performed with 24-well transwell plates according to the manufacturer's instructions (Corning). Briefly, cells were serum-starved overnight, harvested with Accutase (Millipore), and counted. The bottom chambers of transwells were filled with $700 \mu \mathrm{l}$ serum-free media or serum, and 30,000 cells per well were plated in triplicate in $100 \mu \mathrm{l}$ serum-free media in the top chambers. After a 24 -h incubation, nonmigratory cells were removed with a cotton swab, and the migratory cells were fixed with formalin and stained with DAPI. The number of DAPI+ cells were counted and the average number of cells invading or migrating was calculated.

Mmp activity assay. The Mmp activity assay (ab112126, Abcam) was used as per the manufacturer's instructions. Briefly, a $70 \%$ confluent $10 \mathrm{~cm}$ plate of cells was washed with PBS and scraped off in $1 \mathrm{ml} \mathrm{0.5 \%} \mathrm{Triton} \mathrm{X} \mathrm{in} \mathrm{PBS.} \mathrm{Cell} \mathrm{lysate} \mathrm{was}$ incubated in ice for $15 \mathrm{~min}$ with occasional inversions, then centrifuged at 10,000 $\mathrm{rpm}$ at $4{ }^{\circ} \mathrm{C}$ for $10 \mathrm{~min}$. The supernatant was separated and used in the protocol as instructed. Mmps were activated for $40 \mathrm{~min}$ at $37^{\circ} \mathrm{C}$ and fluorescence was read on a Synergy Neo plate reader.

Treatment and MRI of orthotopic allograft mouse models. Allograft of lung adenocarcinoma cells was performed as previously described. Tumor bearing mice were randomized into cohorts treated either with vehicle (Kolliphor-EL) or $55 \mathrm{mg} /$ $\mathrm{kg}$ JIB-04 $3 \times$ per week for 7 weeks. The mice were imaged by magnetic resonance imaging biweekly to determine the tumor volume during the respective treatments as described previously in a non-blinded fashion ${ }^{52}$. The tumor burden volume and quantification were reconstructed on the 3D slicer software (http://www.slicer.org) Imaging was performed with a Nikon 90i camera and NIS-Elements software and processed with NIS-Elements and Adobe Photoshop.

Statistics. The tests used to determine statistical significance are quoted in the appropriate figure legend. $P$ values are quoted either within the text or when indicated in the figure itself, and $P$ values $<0.05$ were considered significant. When comparing sets of continuous data from different animals, the

Kolmogorov-Smirnov test for normalcy was used to determine if a parametric or non-parametric test was required, except where indicated, all tests were two tailed.

\section{Data availability}

Data are available upon reasonable request. The ChIP-seq data and RNA-seq that support the findings of this study have been deposited in NCBI's Gene Expression Omnibus and are accessible through GEO Series accession numbers GSE100455 and GSE120073. Correspondence and requests for materials should be addressed to C.F.K.

Received: 17 August 2018 Accepted: 10 October 2018

Published online: 19 November 2018

\section{References}

1. Curtis, S. J. et al. Primary tumor genotype is an important determinant in identification of lung cancer propagating cells. Cell Stem Cell 7, 127-133 (2010).

2. Lau, A. et al. Tumor-propagating cells and Yap/Taz activity contribute to lung tumor progression and metastasis. EMBO J. 3, 468-481 (2014).

3. Zheng, Y. et al. A rare population of CD24+ITGB4+Notchhi cells drives tumor propagation in NSCLC and requires Notch3 for self-renewal. Cancer Cell 24, 59-74 (2013).

4. de Lera, A. R. \& Ganesan, A. Epigenetic polypharmacology: from combination therapy to multitargeted drugs. Clin. Epigenet. 8, 105 (2016).

5. Jones, P. A., Issa, J.-P. J. \& Baylin, S. Targeting the cancer epigenome for therapy. Nat. Rev. Genet. 17, 630-641 (2016). 
6. Tachibana, M. et al. G9a histone methyltransferase plays a dominant role in euchromatic histone $\mathrm{H} 3$ lysine 9 methylation and is essential for early embryogenesis. Genes Dev. 16, 1779-1791 (2002).

7. Roopra, A., Qazi, R., Schoenike, B., Daley, T. J. \& Morrison, J. F. Localized domains of G9a-mediated histone methylation are required for silencing of neuronal genes. Mol. Cell 14, 727-738 (2004).

8. Casciello, F., Windloch, K., Gannon, F. \& Lee, J. S. Functional role of G9a histone methyltransferase in cancer. Front. Immunol. 6, 487 (2015).

9. Chen, M.-W. et al. H3K9 histone methyltransferase G9a Promotes lung cancer invasion and metastasis by silencing the cell adhesion molecule Ep-CAM. Cancer Res. 70, 7830-7840 (2010).

10. Ding, J. et al. The histone $\mathrm{H} 3$ methyltransferase G9A epigenetically activates the serine-glycine synthesis pathway to sustain cancer cell survival and proliferation. Cell Metab. 18, 896-907 (2013).

11. Dong, C. et al. G9a interacts with Snail and is critical for Snail-mediated Ecadherin repression in human breast cancer. J. Clin. Invest. 122, 1469-1486 (2012).

12. Zhang, J. et al. Down-regulation of G9a triggers DNA damage response and inhibits colorectal cancer cells proliferation. Oncotarget 6, 2917-2927 (2015).

13. Liu, F. et al. Optimization of cellular cctivity of G9a inhibitors 7-aminoalkoxyquinazolines. J. Med. Chem. 54, 6139-6150 (2011).

14. Pappano, W. N. et al. The histone methyltransferase inhibitor A-366 uncovers a role for G9a/GLP in the epigenetics of leukemia. PLoS ONE 10, e0131716 (2015).

15. Yuan, Y. et al. A small-molecule probe of the histone methyltransferase G9a induces cellular senescence in pancreatic adenocarcinoma. ACS Chem. Biol. 7, $1152-1157$ (2012).

16. Yamane, K. et al. JHDM2A, a JmjC-Containing H3K9 demethylase, facilitates transcription activation by androgen receptor. Cell 125, 483-495 (2006).

17. Horton, J. R. et al. Enzymatic and structural insights for substrate specificity of a family of jumonji histone lysine demethylases. Nat. Struct. Mol. Biol. 17, 38-43 (2010).

18. Ma, D. K., Chiang, C.-H. J., Ponnusamy, K., Ming, G.-1 \& Song, H. G9a and Jhdm2a regulate embryonic stem cell fusion-induced reprogramming of adult neural stem cells. Stem Cells 26, 2131-2141 (2008).

19. Shao, P. et al. Histone demethylase PHF8 promotes epithelial to mesenchymal transition and breast tumorigenesis. Nucleic Acids Res. 45, 1687-1702 (2017).

20. Ramadoss, S. et al. Lysine-specific demethylase KDM3A regulates ovarian cancer stemness and chemoresistance. Oncogene 36, 1537-1545 (2017).

21. Vedadi, M. et al. A chemical probe selectively inhibits G9a and GLP methyltransferase activity in cells. Nat. Chem. Biol. 7, 566-574 (2011).

22. Wen, B., Wu, H., Shinkai, Y., Irizarry, R. A. \& Feinberg, A. P. Large histone H3 lysine 9 dimethylated chromatin blocks distinguish differentiated from embryonic stem cells. Nat. Genet. 41, 246-250 (2009).

23. Chen, X. et al. G9a/GLP-dependent histone H3K9me2 patterning during human hematopoietic stem cell lineage commitment. Genes Dev. 26, 2499-2511 (2012).

24. Regala, R. P. et al. Matrix metalloproteinase-10 promotes Kras-mediated bronchio-alveolar stem cell expansion and lung cancer formation. PLoS ONE 6, e26439 (2011)

25. Justilien, V. et al. Matrix metalloproteinase-10 Is required for lung cancer stem cell maintenance, tumor initiation and metastatic potential. PLOS ONE 7, e35040 (2012).

26. Imielinski, M. et al. Mapping the hallmarks of lung adenocarcinoma with massively parallel sequencing. Cell 150, 1107-1120 (2012).

27. Huang, T. et al. G9A promotes tumor cell growth and invasion by silencing CASP1 in non-small-cell lung cancer cells. Cell Death Dis. 8, e2726 (2017).

28. Wang, L. et al. A small molecule modulates Jumonji histone demethylase activity and selectively inhibits cancer growth. Nat. Commun. 4, 2035-2035 (2013).

29. Bai, K. et al. Association of histone methyltransferase G9a and overall survival after liver resection of patients with hepatocellular carcinoma with a median observation of 40 months. Medicine 95, e2493 (2016).

30. Tao, H. et al. Histone methyltransferase G9a and H3K9 dimethylation inhibit the self-renewal of glioma cancer stem cells. Mol. Cell. Biochem. 394, 23-30 (2014).

31. Mariya, T. et al. Matrix metalloproteinase-10 regulates stemness of ovarian cancer stem-like cells by activation of canonical Wnt signaling and can be a target of chemotherapy-resistant ovarian cancer. Oncotarget 7, 26806-26822 (2016).

32. Merchant, N. et al. Matrix metalloproteinases: their functional role in lung cancer. Carcinogenesis 38, 766-780 (2017).

33. Alaseem, A. et al. Matrix metalloproteinases: $\mathrm{q}$ challenging paradigm of cancer management. Semin. Cancer Biol. https://doi.org/10.1016/j.semcancer.2017.11.008 (2017).
34. Yang, H. et al. Elevated JMJD1A is a novel predictor for prognosis and a potential therapeutic target for gastric cancer. Int. J. Clin. Exp. Pathol. 8, 11092-11099 (2015).

35. Dalvi, M. P. et al. Taxane-platin resistant lung cancers co-develop hypersensitivity to JumonjiC demethylase inhibitors. Cell Rep. 19, 1669-1684 (2017).

36. Suzuki, T. et al. Identification of the KDM2/7 histone lysine demethylase subfamily inhibitor and its antiproliferative activity. J. Med. Chem. 56, 7222-7231 (2013).

37. Chu, C.-H. et al. KDM4B as a target for prostate cancer: structural analysis and selective inhibition by a novel inhibitor. J. Med. Chem. 57, 5975-5985 (2014).

38. Fillmore, C. M. et al. EZH2 inhibition sensitizes BRG1 and EGFR mutant lung tumors to TopoII inhibitors. Nature 520, 239-242 (2015).

39. Wang, L. et al. Histone H3K9 methyltransferase G9a represses PPAR $\gamma$ expression and adipogenesis. EMBO J. 32, 45-59 (2013).

40. Wang, L., Jin, Q., Lee, J.-E., Su, I. \& Ge, K. Histone H3K27 methyltransferase Ezh2 represses Wnt genes to facilitate adipogenesis. Proc. Natl Acad. Sci. USA 107, 7317-7322 (2010).

41. Young, N. P. \& Jacks, T. Tissue-specific p19(Arf) regulation dictates the response to oncogenic K-ras. Proc. Natl Acad. Sci. USA 107, 10184-10189 (2010).

42. Gierut, J. J., Jacks, T. E. \& Haigis, K. M. Producing and concentrating LentiCre for mouse infections. Cold Spring Harb. Protoc. 2014, 304-306 (2014).

43. Zhang, Y. et al. Model-based analysis of ChIP-Seq (MACS). Genome Biol. 9, R137-R137 (2008).

44. Robinson, J. T. et al. Integrative Genomics Viewer. Nat. Biotechnol. 29, 24-26 (2011).

45. Dobin, A. et al. STAR: ultrafast universal RNA-seq aligner. Bioinformatics 29, 15-21 (2013).

46. Love, M. I., Huber, W. \& Anders, S. Moderated estimation of fold change and dispersion for RNA-seq data with DESeq2. Genome Biol. 15, 550 (2014)

47. Trapnell, C. et al. Transcript assembly and abundance estimation from RNASeq reveals thousands of new transcripts and switching among isoforms. Nat. Biotechnol. 28, 511-515 (2010).

48. Jackson, E. L. et al. Analysis of lung tumor initiation and progression using conditional expression of oncogenic K-ras. Genes Dev. 15, 3243-3248 (2001).

49. Jackson, E. L. et al. The differential effects of mutant p53 alleles on advanced murine lung cancer. Cancer Res. 65, 10280-10288 (2005).

50. Shedden, K. et al. Gene expression-based survival prediction in lung adenocarcinoma: a multi-site, blinded validation study. Nat. Med. 14, 822-827 (2008).

51. Li, C. \& Wong, W. H. Model-based analysis of oligonucleotide arrays: expression index computation and outlier detection. Proc. Natl Acad. Sci. USA 98, 31-36 (2001).

52. Ji, H. et al. The impact of human EGFR kinase domain mutations on lung tumorigenesis and in vivo sensitivity to EGFR-targeted therapies. Cancer Cell 9, 485-495 (2006)

\section{Acknowledgements}

We thank D. Foster and C. Garcia de Alba Rivas for experimental assistance, the Kim Lab for technical assistance and discussions, L. Zon, K. Cichowski, A. Gutierrez, and F. Camargo for helpful discussions. We also thank R. Mathieu and M. Patinak of the Boston Children's Hospital Flow Cytometry facility for assistance with FACS experiments and the Molecular Biology Core Facility at the Dana-Farber Cancer Institute for assistance with RNA-seq experiments. This work was supported in part by the IASLC Young Investigator Fellowship (S.P.R.), R01 HL090136, R01 HL132266, R01 HL125821, U01 HL100402 RFA-HL-09-004, American Cancer Society Research Scholar Grant RSG-08 082-01-MGO, the V Foundation for Cancer Research, the Thoracic Foundation, the Ellison Foundation, the BCH-Broad Institute Award, The Harvard Medical School Translational Seed Grant and the Harvard Stem Cell Institute (C.F.K.), K22 CA201036 (C.F.B.), American Lung Association and ATS Foundation (H.W.), and the National Cancer Institute R01 CA195740, CA205150, CA166480, CA140594, CA216188, P01 CA154303, and U01 CA213333 (K.K.W.).

\section{Author contributions}

S.P.R. and C.F.K. designed the study; S.P.R., A.F.M.D., S.M.L., B.P.M., P.P., and C.R.A. performed the experiments, C.F.B. analyzed the Director's Challenge data set, S.J.T. and H.W. performed the ChIP-seq experiments and analysis, A.L. and S.R. advised with Mmp and matrix experiments, B.J.A. performed analysis on RNA-seq gene data, F.L. performed the mouse allograft and drug treatments, K.K.W. allowed mouse models studies in his laboratory, T.M.S. provided the chemical library, S.P.R. and C.F.K. wrote the manuscript with comments from all authors.

\section{Additional information}

Supplementary Information accompanies this paper at https://doi.org/10.1038/s41467018-07077-1. 
Competing interests: C.F.K. has a sponsored research agreement with Celgene Corporation and has received honorarium from AstraZeneca and MedImmune. K.K.W. is a founder and equity holder of G1 Therapeutics and he has Consulting/Sponsored Research Agreements with AstraZeneca, Janssen, Pfizer, Array, Novartis, Merck, Takeda, Ono, and BMS. S.P.R., A.F.M.D., S.M.L., B.P.M., P.P., C.R.A., C.F.B., S.J.T., H.W., A.L., S. R., B.J.A., F.L., and T.M.S. have no competing interests.

Reprints and permission information is available online at http://npg.nature.com/ reprintsandpermissions/

Publisher's note: Springer Nature remains neutral with regard to jurisdictional claims in published maps and institutional affiliations. (c) (i) Open Access This article is licensed under a Creative Commons Attribution 4.0 International License, which permits use, sharing, adaptation, distribution and reproduction in any medium or format, as long as you give appropriate credit to the original author(s) and the source, provide a link to the Creative Commons license, and indicate if changes were made. The images or other third party material in this article are included in the article's Creative Commons license, unless indicated otherwise in a credit line to the material. If material is not included in the article's Creative Commons license and your intended use is not permitted by statutory regulation or exceeds the permitted use, you will need to obtain permission directly from the copyright holder. To view a copy of this license, visit http://creativecommons.org/ licenses/by/4.0/.

(C) The Author(s) 2018 